\section{Inflammatory monocytes expressing tissue factor drive SIV and HIV coagulopathy}

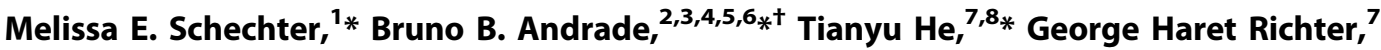 \\ Kevin W. Tosh, ${ }^{2}$ Benjamin B. Policicchio, ${ }^{7,9}$ Amrit Singh, ${ }^{10}$ Kevin D. Raehtz, ${ }^{711}$ Virginia Sheikh, ${ }^{10}$ \\ Dongying Ma, ${ }^{12}$ Egidio Brocca-Cofano, ${ }^{7,8}$ Cristian Apetrei, ${ }^{7,11}$ Russel Tracy, ${ }^{13}$ Ruy M. Ribeiro, ${ }^{14,15}$ \\ Alan Sher, ${ }^{2}$ Ivo M. B. Francischetti, ${ }^{12}$ Ivona Pandrea, ${ }^{7,8+\neq}$ Irini Sereti ${ }^{10+\neq}$
}

In HIV infection, persistent inflammation despite effective antiretroviral therapy is linked to increased risk of noninfectious chronic complications such as cardiovascular and thromboembolic disease. A better understanding of inflammatory and coagulation pathways in HIV infection is needed to optimize clinical care. Markers of monocyte activation and coagulation independently predict morbidity and mortality associated with non-AIDS events. We identified a specific subset of monocytes that express tissue factor (TF), persist after virological suppression, and trigger the coagulation cascade by activating factor $X$. This subset of monocytes expressing TF had a distinct gene signature with up-regulated innate immune markers and evidence of robust production of multiple proinflam-

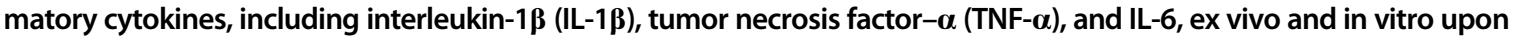
lipopolysaccharide stimulation. We validated our findings in a nonhuman primate model, showing that TF-expressing inflammatory monocytes were associated with simian immunodeficiency virus (SIV)-related coagulopathy in the progressive [pigtail macaques (PTMs)] but not in the nonpathogenic (African green monkeys) SIV infection model. Last, Ixolaris, an anticoagulant that inhibits the TF pathway, was tested and potently blocked functional TF activity in vitro in HIV and SIV infection without affecting monocyte responses to Toll-like receptor stimulation. Strikingly, in vivo treatment of SIV-infected PTMs with Ixolaris was associated with significant decreases in D-dimer and immune activation. These data suggest that TF-expressing monocytes are at the epicenter of inflammation and coagulation in chronic HIV and SIV infection and may represent a potential therapeutic target.
Copyright $\odot 2017$ The Authors, some rights reserved; exclusive licensee American Association for the Advancement of Science. No claim to original U.S. Government Works

\section{INTRODUCTION}

Monocytes are key mediators of innate immunity and have been closely associated with pathogenesis of chronic viral infections, including HIV $(1,2)$. Heightened circulating levels of monocyte activation markers, such as soluble tissue factor (TF), soluble CD14 (sCD14), and soluble CD163 (sCD163), have been associated with increased risk

\footnotetext{
${ }^{1}$ Clinical Research Directorate/Clinical Monitoring Research Program, Leidos Biomedical Research Inc., Frederick National Laboratory for Cancer Research, Frederick, MD 21701, USA. ${ }^{2}$ Immunobiology Section, Laboratory of Parasitic Diseases, National Institute of Allergy and Infectious Diseases, National Institutes of Health, Bethesda, MD 20892, USA. Instituto Gonçalo Moniz, Fundação Oswaldo Cruz, Salvador 40296-710, Brazil. ${ }^{4}$ Multinational Organization Network Sponsoring Translational and Epidemiological Research (MONSTER) Initiative, Instituto Brasileiro para a Investigação da Tuberculose, Fundação José Silveira, Salvador 40210-320, Brazil. ${ }^{5}$ Wellcome Centre for Infectious Disease Research in Africa, Institute of Infectious Disease and Molecular Medicine, University of Cape Town, Cape Town 7925, South Africa. ${ }^{6}$ Division of Infectious Diseases, Department of Medicine, Vanderbilt University School of Medicine, Nashville, TN 37232, USA. ${ }^{7}$ Center for Vaccine Research, School of Medicine, University of Pittsburgh, Pittsburgh, PA 15261, USA. ${ }^{8}$ Department of Pathology, School of Medicine, University of Pittsburgh, Pittsburgh, PA 15261, USA. ${ }^{9}$ Department of Infectious Diseases and Microbiology, Graduate School of Public Health, University of Pittsburgh, Pittsburgh, PA 15261, USA. ${ }^{10} \mathrm{HIV}$ Pathogenesis Section, Laboratory of Immunoregulation, National Institute of Allergy and Infectious Diseases, National Institutes of Health, Bethesda, MD 20892, USA. "1 Department of Microbiology and Molecular Genetics, School of Medicine, University of Pittsburgh, Pittsburgh, PA 15261, USA. ${ }^{12}$ Laboratory of Malaria and Vector Research, National Institute of Allergy and Infectious Diseases, National Institutes of Health, Bethesda, MD 20892, USA. ${ }^{13}$ Department of Pathology and Laboratory Medicine, The Robert Larner, M.D. College of Medicine University of Vermont, Burlington, VT 05405, USA. ${ }^{14}$ Theoretical Biology and Biophysics, Los Alamos National Laboratory, Los Alamos, NM 87544, USA. ${ }^{15}$ Laboratório de Biomatemática, Faculdade de Medicina, Universidade de Lisboa, Lisboa 1649-028, Portugal.

*These authors contributed equally to this work.

†Corresponding author. Email: bruno.andrade@bahia.fiocruz.br (B.B.A.); isereti@ niaid.nih.gov (I.S.); pandrea@pitt.edu (I.P.)

‡These authors contributed equally to this work.
}

for death (3), noninfectious complications $(4,5)$, subclinical atherosclerosis (6), and immune reconstitution inflammatory syndrome (IRIS) in HIV-infected individuals (7). Moreover, differential activation of monocyte subsets has recently been described as a predictor of tuberculosis (TB)-associated IRIS in patients with HIV-TB co-infection (7). One important feature of monocytes in HIV pathogenesis is their capacity to produce TF $(8-10)$. TF is expressed in response to inflammatory stimuli, such as Toll-like receptors (TLRs) (11-13) and cytokinedriven signals $(14,15)$, and initiates the extrinsic coagulation cascade by cleaving coagulation factors, leading to the formation of factor Xa, thrombin, and fibrin, which, when degraded, forms the coagulation biomarker D-dimer $(16,17)$. For these reasons, augmented TF expression is associated with increased levels of D-dimer (18) and thus may be associated with an increased risk for cardiovascular complications in HIV-infected individuals (19). These findings support a direct role of activated monocytes in the persistent inflammatory milieu observed in chronic HIV infection.

The need to investigate the link between coagulation and inflammation in chronic viral infections is pressing. Inflammatory and coagulation markers are both independent predictors of morbidity and mortality in treated HIV individuals (20-23) and are associated with noninfectious complications of HIV, such as cardiovascular and thromboembolic disease (19), which are rising due to the aging of treated HIV-infected persons (24). In an experimental model of nonhuman primates (NHPs) infected with SIVsab, we previously demonstrated that increases in D-dimer and monocyte activation markers (sCD14) predict disease progression (25). These findings highlighted monocyte activation as a key event driving persistent coagulation in simian immunodeficiency virus (SIV)/HIV chronic infection, suggesting a need to delineate the role of monocyte-derived TF in SIV/HIV-driven systemic inflammation and coagulopathy. 
Here, we evaluated the role of TF-expressing monocytes in HIV and SIV pathogenesis and related coagulopathy. We examined the links between inflammation and coagulation with the aim to identify potential targets for therapeutic interventions in HIV-infected persons. activation and coagulation using a multiplex quantitative polymerase chain reaction (qPCR) assay. Hierarchical clustering analysis of the overall gene expression profile of monocytes revealed that $\mathrm{TF}^{\mathrm{pos}}$ cells exhibit a unique signature highlighted by increased expression of
RESULTS

\section{A specific monocyte subset is the major source of TF among peripheral blood mononuclear cells}

To determine the potential of monocytes to express TF, we stimulated peripheral blood mononuclear cells (PBMC) from healthy blood donors with lipopolysaccharide (LPS) in vitro, and we assessed TF expression by flow cytometry (monocyte gating strategy shown in fig. S1). The frequency of monocytes expressing $\mathrm{TF}$ [TF-positive $\left(\mathrm{TF}^{\mathrm{pos}}\right)$ ] in unstimulated cultures was very low [median, $0.49 \%$ of total monocytes; interquartile range (IQR), 0.24 to 0.69 ]. Upon LPS stimulation, the expression of TF by monocytes was significantly increased $(P<0.0001$; Fig. 1A). We next tested whether TF expression reflects a state of cellular activation by quantifying the frequency of $\mathrm{TF}^{\mathrm{pos}}$ monocytes after stimulation with increasing doses of LPS. A robust expression of TF by monocytes was observed only when cells were stimulated with LPS ( $\geq 10 \mathrm{ng} / \mathrm{ml}$ ), whereas lower doses triggered a minor induction of the enzyme (Fig. 1B). The frequency of $\mathrm{TF}^{\mathrm{pos}}$ monocytes did not increase further with escalated doses of LPS (Fig. 1B), suggesting that only a subset of cells is capable of producing the coagulation factor in vitro. We next performed a phenotypic analysis to better delineate the subpopulation of mononuclear cells from PBMC that produce TF upon LPS stimulation. We observed that TF expression is restricted to HLA-DR $^{+}$, Dump ${ }^{-}\left(\mathrm{CD}^{-} \mathrm{CD}^{-} \mathrm{CD}^{-} 9^{-}\right.$ $\mathrm{CD} 20^{-} \mathrm{CD}^{-} 6^{-}$) cells, which were further characterized as $\mathrm{CD} 14^{+}, \mathrm{CD} 16^{-}$, and $\mathrm{CCR}^{+}$monocytes (fig. S2). A more detailed analysis revealed that $\mathrm{TF}^{\mathrm{pos}}$ cells exhibit differential expression of activation markers and costimulatory molecules, such as CD36, CD40, CD86, CD62L, CD163, TLR4, and interleukin-6 receptor (IL-6R) (fig. S2). To further investigate these populations, we sorted $\mathrm{TF}^{\mathrm{pos}}$ and $\mathrm{TF}^{\text {neg }}$ monocytes from six healthy donors after LPS stimulation and examined the expression profile of a customized assortment of genes associated with monocyte
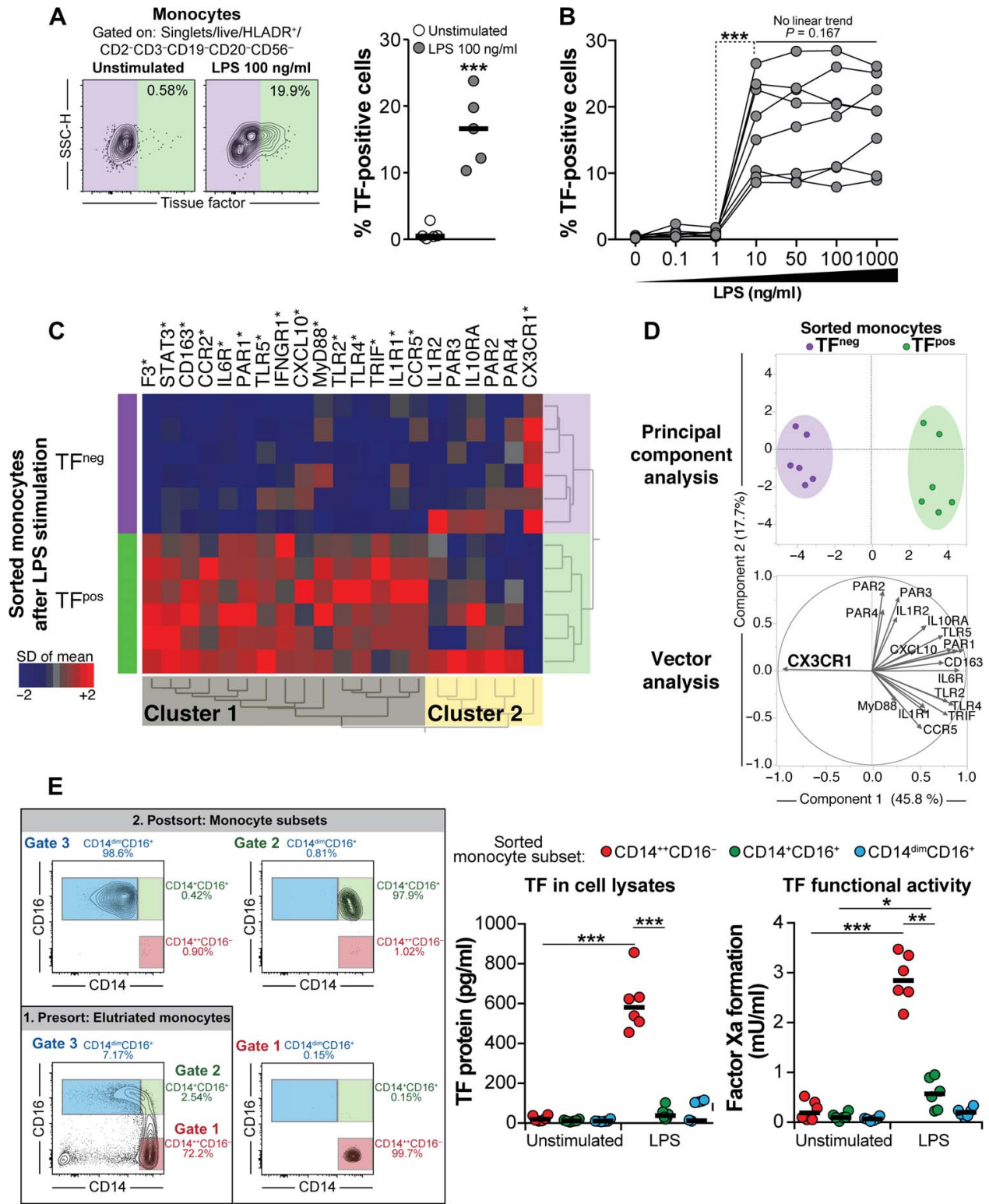

Fig. 1. LPS drives TF expression on human monocyte subsets. (A) Left: Representative fluorescence-activated cell sorting plots of TF expression on human monocytes from healthy controls upon LPS stimulation in vitro. Right: Summary data $(n=5)$ of frequency of $\mathrm{TF}^{\text {pos }}$ monocytes. Lines represent median values. Data were analyzed using Mann-Whitney $U$ test. SSC-H, side-scatter height. (B) Frequency of TF-expressing monocytes on PBMC from healthy controls stimulated with increasing doses of LPS in vitro $(n=8)$. Data were analyzed using Kruskal-Wallis test with Dunn's multiple comparisons and linear trend ad hoc test. (C) Hierarchical cluster analysis of the expression profile ( $z$-score-normalized) of indicated genes assessed by qPCR in monocytes ( $n=6$ healthy donors) sorted after 6 hours of LPS stimulation (100 ng/ml), as described in Materials and Methods. (D) PCA of the expression level of indicated genes was performed. (E) Monocyte subsets were sorted on the basis of surface expression of CD14 and CD16 ( $n=6$ healthy donors). Representative plots show monocytes before and after sorting (left). TF protein expression in cell lysates and TF functional activity measured by the formation of factor $\mathrm{Xa}$ in vitro were compared between the different monocyte subsets in vitro using Kruskal-Wallis test with Dunn's multiple comparisons posttest. Lines represent median values. ${ }^{*} P<0.05,{ }^{* *} P<0.01,{ }^{* * *} P<0.001$. 
TLRs, proinflammatory cytokine receptors, and signaling molecules such as MyD88 and TRIF (Fig. 1, C and D), whereas TF ${ }^{\text {neg }}$ cells exhibited increased relative expression of CX3CR1 (Fig. 1, C and D). Principal component analysis (PCA) with vector analysis of the expression profile of all genes confirmed the observation that $\mathrm{TF}^{\mathrm{pos}}$ and $\mathrm{TF}^{\text {neg }}$ monocytes have distinct gene expression profiles associated with inflammation and coagulation (Fig. 1D). These findings indicate that rather than representing a general state of cell activation, TF expression by circulating mononuclear cells may be restricted to a specific monocyte subset.

It is known that expression of surface markers on monocytes is dynamic and can change depending on the stimulation and microenvironment conditions (26). To address whether this dynamic expression of surface markers was confounding our interpretation of which monocyte subset expresses TF in response to LPS, we sorted the three major monocyte subsets based on the dichotomous surface expression of CD14 and CD $16\left(\mathrm{CD} 14^{++} \mathrm{CD} 16^{-}, \mathrm{CD} 14^{+} \mathrm{CD} 16^{+}\right.$, and $\left.\mathrm{CD} 14^{\mathrm{dim}} \mathrm{CD} 16^{+}\right)$, stimulated each subset with LPS, and then examined TF expression in cell lysates and measured TF functional activity by a colorimetric assay, which quantifies the formation of the coagulation factor Xa (Fig. 1E) (27). Under unstimulated conditions, TF protein expression and functional activity by each monocyte subset were uniformly low in cultures (Fig. 1E). LPS stimulation induced an increase in TF protein expression and activity in $\mathrm{CD} 14^{++} \mathrm{CD} 16^{-}$monocytes, a marginal increase of TF functional activity (by means of factor Xa formation) in $\mathrm{CD} 14^{+} \mathrm{CD} 16^{+}$cells, and no effect on $\mathrm{CD} 14^{\mathrm{dim}} \mathrm{CD} 16^{+}$monocytes (Fig. 1E). These data demonstrate an inherent capacity for the $\mathrm{CD} 14^{++} \mathrm{CD} 16^{-}$ monocyte subset to express TF after LPS-driven activation.

\section{TF-expressing monocytes are expanded in chronic HIV infection independent of antiretroviral treatment}

It has been previously observed that monocytes from $\mathrm{HIV}^{+}$patients display higher levels of TF expression compared to healthy controls (18). Here, we extended these observations by comparing TF expression in $\mathrm{HIV}^{+}$individuals before and after antiretroviral therapy (ART) initiation (after virological suppression was achieved) and age- and gendermatched healthy controls (table S2). In a cross-sectional comparison of ex vivo TF expression, measured by flow cytometry, we observed that the frequency of $\mathrm{TF}^{\mathrm{pos}}$ monocytes was significantly higher in both ARTnaïve and ART-treated $\mathrm{HIV}^{+}$patients compared to healthy controls $(P<$ 0.001 and $P<0.01$, respectively; Fig. $2 \mathrm{~A}$ ), albeit with considerable variability in expression levels. The median frequency of $\mathrm{TF}^{\mathrm{pos}}$ cells was not statistically different between treatment-naïve $\mathrm{HIV}^{+}$patients and those with ART-induced suppression of HIV viremia (Fig. 2A). This finding suggests that TF protein expression in $\mathrm{HIV}^{+}$patients may not be substantially affected by ART. We next compared the potential of monocytes to produce TF in response to LPS between the different study groups. We found that the frequencies of $\mathrm{TF}^{\mathrm{pos}}$ monocytes were significantly higher in $\mathrm{HIV}^{+}$patients, before and after ART, compared to healthy individuals in unstimulated cultures as well as upon LPS stimulation (Fig. 2B). There was no observed difference in response to LPS between treatment-naïve $\mathrm{HIV}^{+}$patients and those on ART with virological suppression (Fig. 2B). These results mirrored the findings obtained by quantification of TF protein expression in PBMC lysates (Fig. 2C). We further demonstrated that TF expressed on the cell surface of monocytes from $\mathrm{HIV}^{+}$individuals was able to trigger factor Xa formation, demonstrating that TF was functionally active in vitro (Fig. 2D). Again, no difference was detected in
A

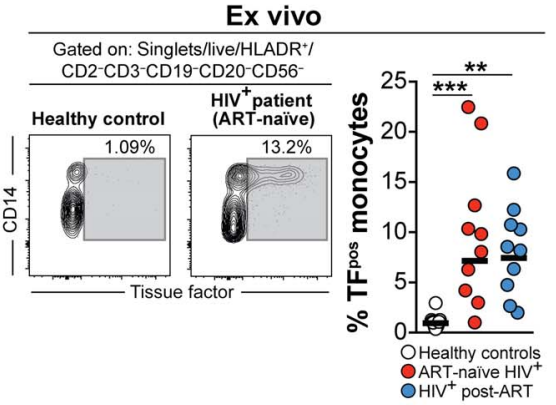

E

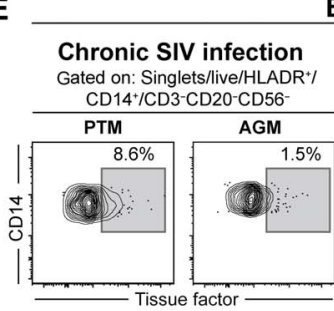

Ex vivo

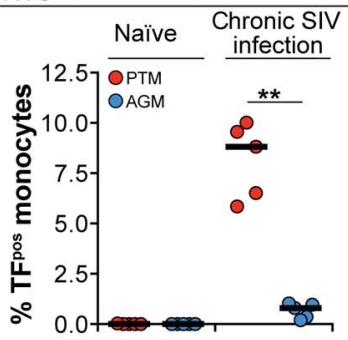

B In vitro

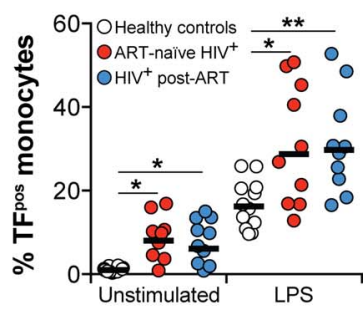

$\mathbf{F}$

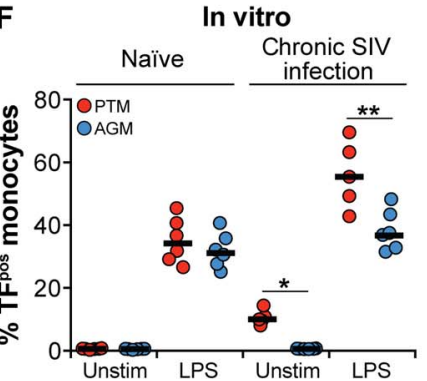

C

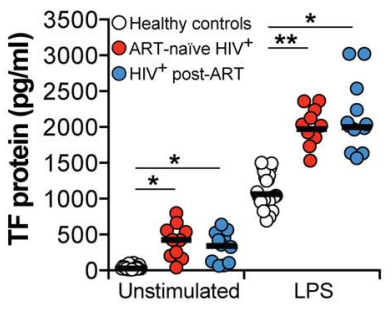

\section{TF functional activity}

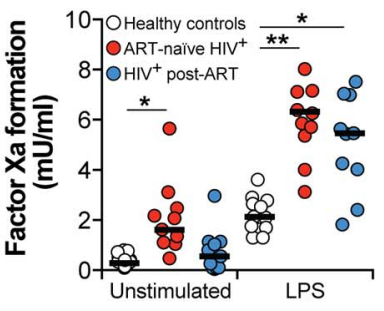

G

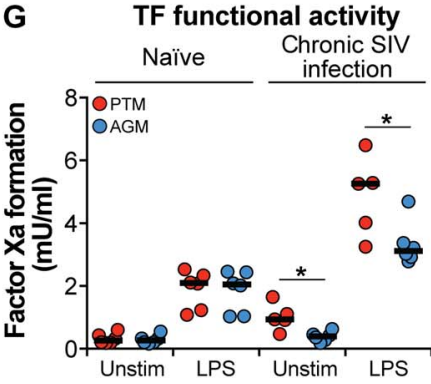

Fig. 2. Frequency of TF-expressing monocytes is increased in chronically HIV-infected individuals and in SIV-infected NHPs despite virological suppression status. (A) Left: Representative plots of TF ${ }^{\text {pos }}$ monocytes ex vivo in healthy controls and HIV $^{+}$patients. Right: Summary data of frequency of TF ${ }^{\text {pos }}$ monocytes from a cross-sectional analysis including healthy controls $(n=12)$, ART-naïve $\mathrm{HIV}^{+}$patients $(n=10)$, and $\mathrm{HIV}^{+}$individuals after ART-induced virological suppression (HIV ${ }^{+}$postART; $n=10$ ). PBMC were stimulated with LPS in vitro, and frequency of TF ${ }^{\text {pos }}$ monocytes (B), TF protein expression in cell lysates (C), and TF functional activity (D) were compared between the cross-sectional study groups using Kruskal-Wallis test with Dunn's multiple comparisons posttest. (E) Left: Representative plots of TF expression on monocytes ex vivo in chronically SIV-infected pigtail macaque (PTMs) $(n=6)$ and African green monkey (AGMs) $(n=6)$. Right: Summary data of frequency of TF ${ }^{\text {pos }}$ monocytes ex vivo from both naïve and chronically SIV-infected animals. PBMC were stimulated with LPS in vitro, and frequency of TF ${ }^{\text {pos }}$ monocytes (F) and TF functional activity (G) were compared between naïve or SIV-infected PTMs and AGMs. Lines represent median values. Data were analyzed using Mann-Whitney $U$ test. ${ }^{*} P<0.05,{ }^{* *} P<0.01,{ }^{* * *} P<0.001$ 
TF functional activity between the groups of $\mathrm{HIV}^{+}$patients (Fig. 2, C and $\mathrm{D})$. The differences in TF expression on monocytes were independent of the total monocyte counts in PBMC among the HIVinfected groups, which were not significantly different (median, 4451 cells/ $\mu \mathrm{l}$; IQR, 2383 to 6829 in ART-naïve versus 4507 cells/ $\mu \mathrm{l}$; IQR, 3308 to 7771 in virologically suppressed individuals; $P=0.34$ ).

These primary observations indicated an increase in the capacity of monocytes to produce TF and promote factor Xa formation in vitro upon cellular activation in HIV infection, which persists after ARTinduced HIV suppression. To further test this hypothesis, we prospectively assessed ex vivo TF expression as well as plasma levels of D-dimer and C-reactive protein (CRP) in an additional set of $\mathrm{HIV}^{+}$patients examined longitudinally with paired samples available from pre- and post-ART (at virological suppression) time points. This longitudinal paired analysis confirmed that the frequency of $\mathrm{TF}^{\mathrm{pos}}$ monocytes was not significantly altered by ART (fig. S3). Of $12 \mathrm{HIV}^{+}$patients prospectively evaluated, 6 (50\%) exhibited increases and 4 (33.3\%) displayed reduction in TF expression upon ART-induced HIV suppression. Mean fluorescent intensity (MFI) values for TF expression did not differ between the time points [MFI at pre-ART, $575 \pm 49.5$ arbitrary units (AU) versus MFI at virologic suppression time point, $553 \pm 89.6$ AU; $P=0.785]$. However, D-dimer levels decreased, whereas CRP values remained unchanged after ART initiation (fig. S3). These findings show sustained elevation of TF expression on circulating monocytes regardless of the reduction in D-dimer levels, which strongly argues that HIV-associated coagulopathy may persist even after ARTinduced HIV suppression, as has been previously suggested (28). In ART-naïve patients, the ex vivo frequency of $\mathrm{TF}^{\mathrm{pos}}$ monocytes was positively correlated with the levels of D-dimer $(r=0.69, P=0.015$; fig. S3) and did not associate with concentrations of CRP $(r=0.04, P=$ 0.908; fig. S3).

To further assess the role of TF on monocytes in HIV pathogenesis and coagulopathy, we next examined the expression and activity of this coagulation factor in PBMCs from NHPs before and after SIVsab infection in vivo. In this biological system, AGMs are the natural hosts of SIV sab and undergo infection but do not progress to SIV disease and coagulopathy despite active chronic viral replication reflected by lifelong high plasma SIV viremia $(29,30)$. Contrastingly, SIV sab infection causes progressive disease in PTMs (29). We have previously shown that PTMs infected with SIVsab experience increased systemic inflammation and coagulopathy compared to naïve animals (25). Before SIVsab infection, both PTMs and AGMs exhibited similarly low frequencies of $\mathrm{TF}^{\text {pos }}$ monocytes (Fig. 2E). The frequency of monocytes expressing TF ex vivo was substantially increased in chronically infected PTMs compared to naïve animals $(P<0.001)$, whereas there was no marked effect of SIV infection in AGMs (Fig. 2E). Monocytes, but not myeloid dendritic cells, were the major source of TF ex vivo and after LPS stimulation in vitro (fig. S4). Notably, monocytes isolated from naïve PTMs and AGMs displayed indistinguishable TF expression in response to LPS stimulation in vitro (Fig. 2F). During chronic SIV sab infection, the frequency of $\mathrm{TF}^{\text {pos }}$ monocytes was higher in PTMs compared to AGMs in unstimulated cells, and this difference was further increased upon LPS stimulation (Fig. 2F). Comparable results were obtained by quantification of TF functional activity (Fig. 2G). In addition, we found in our cohort of NHPs that concentrations of D-dimer and CRP in plasma were significantly higher in accordance with the augmented TF monocyte expression in chronically infected PTMs compared to naïve animals (table S1). Neither TF expression nor levels of these plasma markers of inflam- mation and coagulation were significantly altered upon SIVsab infection in AGMs (table S1). To further investigate the role of TF in SIV pathogenesis, we examined TF expression before infection and at different time points after infection in the mucosal tissues sampled from PTMs. We observed a continuous increase of TF expression in the gut with disease progression (fig. S5). Furthermore, few $\mathrm{TF}^{\mathrm{pos}}$ cells were present in the lamina propria before infection, but their numbers increased after infection, first at the tip of the villi and then more profoundly in the lamina propria. The same dynamics were also observed in Peyer's patches (fig. S5). The significant increase in TF expression of both mucosal tissues and peripheral monocytes of chronically SIVinfected PTM suggests that TF expression may contribute to the pathogenesis of SIV-driven persistent coagulation and inflammation.

\section{Proinflammatory cytokines increase TF expression during chronic HIV infection}

A potential explanation for the activation of circulating monocytes and subsequent persistent TF expression in HIV-infected persons could be a soluble factor. To test this hypothesis, we column-purified CD $14^{+}$ monocytes from healthy blood donors and incubated them overnight with medium supplemented with either heat-inactivated serum from human $\mathrm{AB}$ plasma (HAB serum), filtered pooled sera from healthy controls, or sera from individuals chronically infected with HIV or hepatitis $\mathrm{C}$ virus (HCV). TF protein expression was then quantified in cell lysates. Samples incubated with $\mathrm{HIV}^{+}$or $\mathrm{HCV}^{+}$sera displayed augmented production of TF (Fig. 3A). TF expression was indistinguishable between cultures of monocytes incubated with sera from ART-naïve $\mathrm{HIV}^{+}$patients and at different time points upon treatment initiation (Fig. 3B). This observation indicates that the factors driving TF expression by monocytes persist after ART implementation. Moreover, we performed a series of blocking experiments trying to delineate the molecules that are potentially driving monocyte activation and TF production from the sera of HIV-infected individuals. We found that IL-1RI blocking resulted in a slight but consistent reduction of TF production in monocytes cultured in the presence of $\mathrm{HIV}^{+}$serum (Fig. 3C). Neutralizing IL-6R and interferon- $\gamma$ receptor (IFN $\gamma \mathrm{R})$ did not alter TF expression in this experimental setting (Fig. 3C). Notably, TF production exhibited a substantial drop in monocyte cultures incubated with blocking antibodies against type I IFN receptor (IFNAR) or tumor necrosis factor receptor 1 (TNFR1) $(P=0.003$ and $P=0.005$, respectively; Fig. $3 \mathrm{C}$ ). These experiments confirmed that inflammatory signals present in serum from $\mathrm{HIV}$-infected persons are capable of inducing $\mathrm{TF}$ production in monocytes. The specific drivers of these inflammatory signals could be coming from inflamed tissues or the blood itself. Microbial translocation in the gut has been described as an important trigger of persistent systemic inflammation detected in $\mathrm{HIV}^{+}$persons (31). To test the possibility that monocyte activation in the blood could result from a residual leakage of microbial products from mucosal interfaces, we incubated $\mathrm{HIV}^{+}$serum with polymyxin B before culturing the monocytes. Serum treated with this bactericidal compound resulted in the lowest TF expression among all the experimental conditions tested (Fig. 3C). These findings demonstrate that circulating microbial products are relevant drivers of TF expression by activated monocytes.

\section{TF-expressing monocytes produce multiple proinflammatory cytokines}

Chronic HIV infection has been associated with persistent immune activation and elevated markers of coagulation $(22,32)$. We hypothesized 

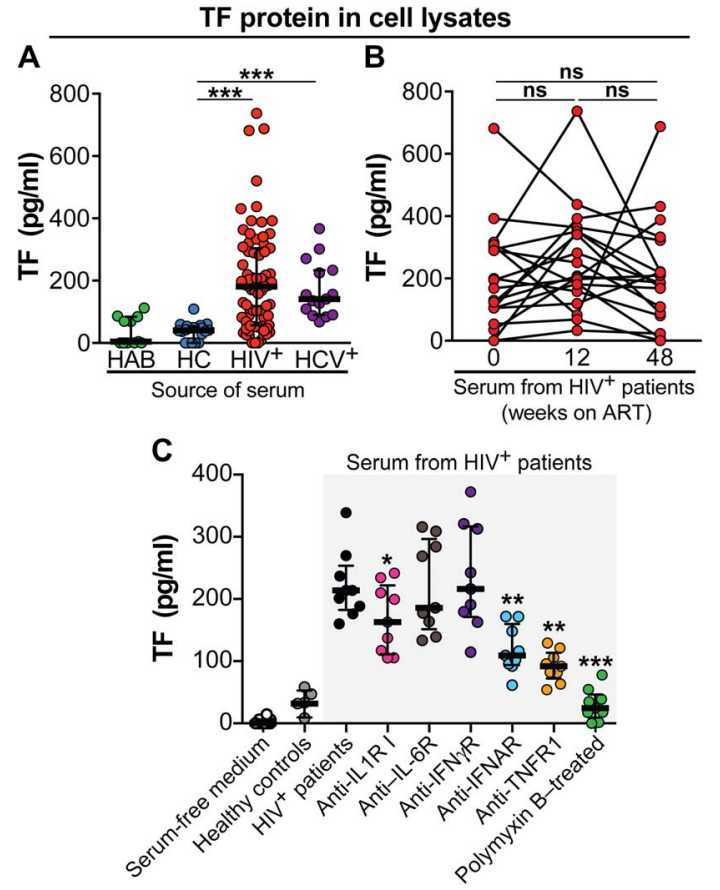

Fig. 3. Inflammatory mediators and microbial products may drive TF expression by circulating monocytes in the context of HIV infection. (A) Columnpurified $\mathrm{CD}_{1} 4^{+}$monocytes from 10 healthy controls were cultured for 18 hours in the presence of RPMl supplemented with $10 \%$ manufactured human $A B$ serum or serum isolated from healthy controls, ART-naïve $\mathrm{HIV}^{+}$patients, or $\mathrm{HCV}^{+}$individuals, as described in Materials and Methods. (B) Monocytes were also cultured in the presence of serum from ART-naïve HIV-infected patients at different time points after ART initiation. (C) Monocytes were cultured for 18 hours in the presence of indicated blocking antibodies $(10 \mu \mathrm{g} / \mathrm{ml})$ or with serum previously treated with polymyxin B $(0.5 \mu \mathrm{g} / \mu \mathrm{l})$. Cells were washed and lysed for the assessment of TF protein expression using enzyme-linked immunosorbent assay. Unmatched data were analyzed using Mann-Whitney $U$ test, whereas matched pairs were compared using Wilcoxon matched pairs test. ${ }^{*} P<0.05$, ${ }^{* *} P<0.01,{ }^{* * *} P<$ 0.001. ns, nonsignificant.

that TF-expressing monocytes, aside from promoting coagulation, could contribute to systemic inflammation. In non-HIV-infected healthy individuals, $\mathrm{TF}^{\mathrm{pos}}$ monocytes more frequently produced multiple proinflammatory cytokines, such as TNF- $\alpha$, IL-1 $\beta$, and IL-6, simultaneously upon LPS stimulation (Fig. 4, A to C). We further investigated the profile of intracellular cytokine expression in monocytes ex vivo from our longitudinal cohort of $\mathrm{HIV}^{+}$patients before ART initiation and after virological suppression (table S3). $\mathrm{HIV}^{+}$individuals, regardless of treatment status, exhibited high frequencies of monocytes spontaneously producing proinflammatory cytokines (fig. S6A). Furthermore, by testing the monocyte response to LPS stimulation in our prospective cohort of $\mathrm{HIV}^{+}$patients, we detected markedly different intracellular cytokine expression profiles between $\mathrm{TF}^{\text {neg }}$ and $\mathrm{TF}^{\mathrm{pos}}$ monocytes in ART-naïve $\mathrm{HIV}^{+}$patients $\left(\chi^{2}, P<0.001\right)$. In contrast to monocytes from healthy controls (Fig. 4C), after LPS stimulation, the vast majority of $\mathrm{TF}^{\text {neg }}$ monocytes from HIV-infected individuals produced IL- 6 alone (median, $48.7 \%$; IQR, 33.5 to $52.8 \%$ of all the cytokine-producing $\mathrm{TF}^{\text {neg }}$ cells), whereas $\mathrm{TF}^{\text {pos }}$ monocytes more frequently produced TNF- $\alpha$, IL- $1 \beta$, and IL- 6 simultaneously ( $53.1 \%$ of all the cytokine-producing $\mathrm{TF}^{\text {pos }}$ cells; IQR, 49.5 to 56.7\%) (Fig. 4D). The intracellular cytokine expression profile of LPS-stimulated monocytes significantly changed after ART initiation $\left(\chi^{2}, P=\right.$
0.015 for the expression profile in $\mathrm{TF}^{\text {neg }}$ cells and $P<0.001$ for $^{\mathrm{T} F} \mathrm{~F}^{\mathrm{pos}}$ monocytes from pre-ART versus post-ART initiation; Fig. 4D). In virologically suppressed $\mathrm{HIV}^{+}$individuals, $\mathrm{TF}^{\text {neg }}$ cells exhibited a mixed cytokine expression profile with monocytes producing one or various combinations of two cytokines, whereas most of the $\mathrm{TF}^{\mathrm{pos}}$ monocytes remained polyfunctional, producing a combination of three cytokines (TNF- $\alpha$, IL-1 $\beta$, and IL- 6 ) simultaneously $\left(\chi^{2}, P<\right.$ 0.001 profile of $\mathrm{TF}^{\text {neg }}$ versus $\mathrm{TF}^{\text {pos }}$ cells; Fig. $4 \mathrm{D}$ ). In addition, the frequency of TF-expressing monocytes strongly correlated with that of polyfunctional monocytes before ART initiation $(r=0.89, P<0.001)$ and in patients with virological suppression $(r=0.91, P<0.001)$.

We next examined the monocyte response to LPS in our NHP cohort by assessing the intracellular expression of TNF- $\alpha$, IL-1 $\beta$, and IL-6 in vitro and found that monocytes isolated from both naïve and chronically SIVsab-infected PTMs and AGMs were capable of producing these cytokines upon LPS stimulation (fig. S6B). The cytokine expression profile of stimulated monocytes was not different between uninfected PTMs and AGMs, with most of the cytokineproducing cells expressing IL- $1 \beta$ alone in both species $\left(\chi^{2}, P=0.459\right.$; Fig. $4 \mathrm{E}$ and fig. S6B). The overall frequency of monocytes simultaneously expressing multiple proinflammatory cytokines (TNF- $\alpha$, IL-1 $\beta$, and IL-6) ex vivo was significantly higher in chronically infected PTMs than in AGMs (Fig. 4F). Upon activation in vitro, the frequency of cytokine-producing monocytes was higher in PTMs than in AGMs (fig. S6B). SIVsab infection caused changes in the cytokine expression profile of stimulated monocytes from both NHP species $\left(\chi^{2}, P=0.035\right.$ for AGM naïve versus chronically infected, $P<0.001$ for PTM naïve versus chronically infected; Fig. $4 \mathrm{E}$ ). During chronic SIVsab infection, most of the activated monocytes from PTMs produced TNF- $\alpha$, IL-1 $1 \beta$, and IL- 6 simultaneously (median, $43.5 \%$ of all the cytokine-producing monocytes; IQR, 35.2 to $55.8 \%$ ), whereas the activated monocytes from AGMs had a mixed expression profile and a low frequency of triple cytokine producers (median, $12.5 \%$ of all the cytokine-producing monocytes; IQR, 8.5 to $18.9 \% ; P<0.001$ versus frequency of triple producers from PTMs) (Fig. 4E).

We next quantitatively compared the dynamics of monocyte polyfunctionality in HIV-infected patients as well as in SIVab-infected NHPs. In $\mathrm{HIV}^{+}$patients, the polyfunctionality of $\mathrm{TF}^{\mathrm{pos}}$ monocytes remained high after ART initiation and was significantly increased in $\mathrm{TF}^{\text {neg }}$ cells after ART compared to $\mathrm{TF}^{\text {neg }}$ cells before ART initiation $(P<0.01$; Fig. $4 \mathrm{~F})$. The polyfunctionality of $\mathrm{TF}^{\mathrm{pos}}$ monocytes from chronically SIVsab ${ }^{+}$PTMs was significantly higher when compared to $\mathrm{TF}^{\text {neg }}$ monocytes from the same animals and also when compared to $\mathrm{TF}^{\text {pos }}$ monocytes isolated from chronically SIVsab ${ }^{+}$AGMs $(P<$ 0.001; Fig. 4G). These findings from both HIV-infected patients and SIV-infected NHPs support the hypothesis that, in monocytes, increased TF expression is associated with an enhanced potential to produce multiple proinflammatory cytokines.

\section{Thrombin triggers TF expression on CD14 ${ }^{\text {high }}$ monocytes and induces production of multiple proinflammatory cytokines via PAR-1 signaling}

Our results so far demonstrated that $\mathrm{CD} 14^{\text {high }}$ monocytes have increased capacity both to promote activation of coagulation factors and to produce multiple proinflammatory cytokines linked to HIV and SIV pathogenesis. Activation of the coagulation cascade by TF in vivo ultimately results in the formation of thrombin, an essential protein leading to thrombus development (33). Thrombin has been shown to activate nuclear factor $\mathrm{\kappa B}(\mathrm{NF}-\mathrm{\kappa B})$ via cleavage of the cytoplasmic tail of 
Fig. 4. TF-expressing monocytes produce multiple proinflammatory cytokines. (A) Representative plots show intracellular cytokine staining for IL-1 $\beta$, IL- 6 , and TNF- $\alpha$ in monocytes from healthy donors ( $n=12$ ) upon LPS stimulation in vitro. SSC-A, side-scatter area. (B) Polyfunctional analysis of TF ${ }^{\text {neg }}$ and TF ${ }^{\text {pos }}$ monocytes upon LPS stimulation. (C to $\mathbf{E})$ The cytokine expression profiles in $\mathrm{TF}^{\text {neg }}$ and $\mathrm{TF}^{\text {pos }}$ monocytes were compared using $\chi^{2}$ tests. (F) Frequency of monocytes producing more than one cytokine ex vivo was compared between TF $^{\text {neg }}$ and TF $^{\text {pos }}$ monocytes in a prospective cohort of ART-naïve $\mathrm{HN}^{+}$patients $(n=15)$ before therapy initiation and after ARTinduced virological suppression ( $\mathrm{HIV}^{+}$post-ART). (G) Frequency of monocytes producing more than one cytokine ex vivo was compared between TF $^{\text {neg }}$ and $\mathrm{TF}^{\mathrm{pos}}$ monocytes in chronically SIV-infected PTMs $(n=6)$ and AGMs $(n=6)$. Unmatched data were compared using the Mann-Whitney $U$ test, whereas matched comparisons were performed using the Wilcoxon matched pairs test. ${ }^{*} P<0.01$, ${ }^{* * *} P<0.001$.

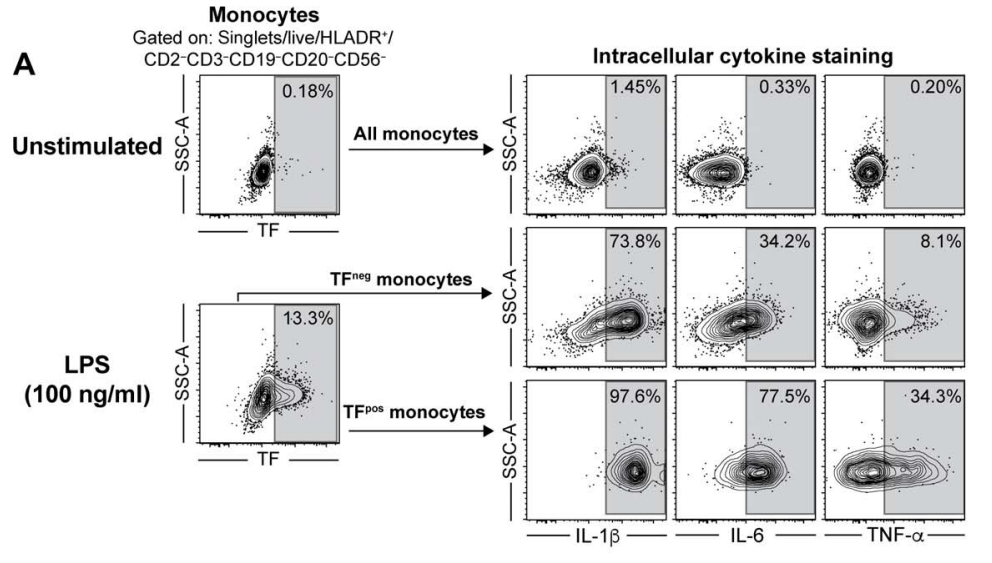

B

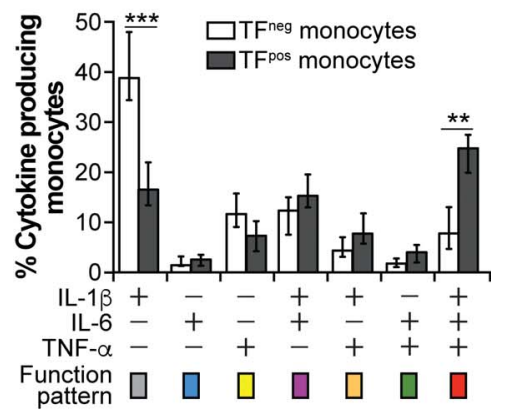

E

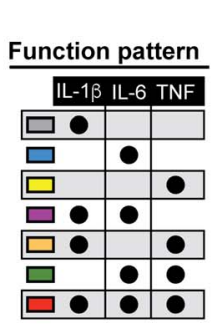

C

D

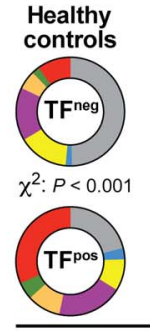

ART-naïve HIV ${ }^{+}$

HIV+ post-ART

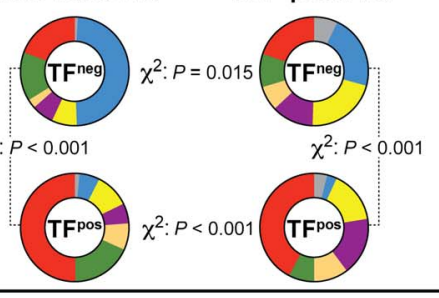

Nonhuman primates

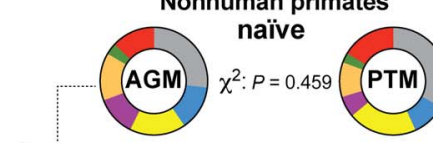

$\chi^{2}: P=0.035$ Chronic SIV infection $\quad \chi^{2}: p<0.001$

Intracellular cytokine expression profile

(LPS-stimulated cells)
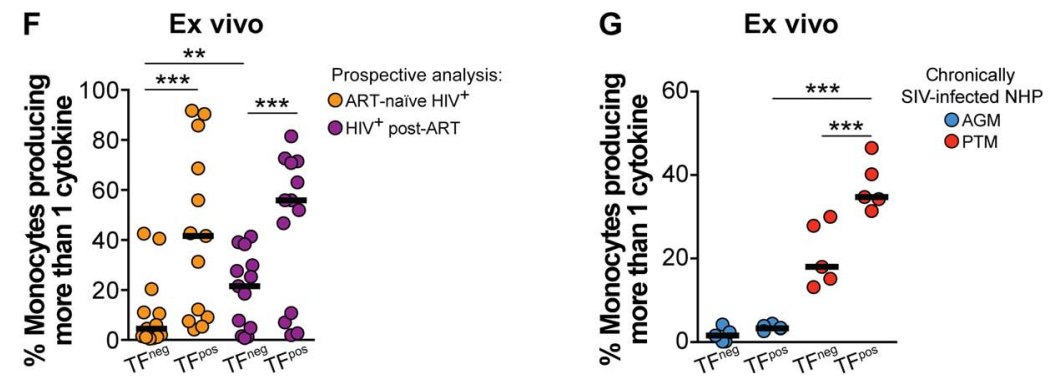

These results led us to speculate that monocytes activated by throm-

protease-activated receptors (PARs) (34). Among the various types of PARs, PAR-1 and PAR-3 are known to be expressed on monocytes (35). A recent study has demonstrated that thrombin triggers TF expression on human monocytes via activation of PAR-1 $(36,37)$. We postulated that activated $\mathrm{CD} 14^{\text {high }} \mathrm{TF}^{\mathrm{pos}}$ monocytes might also be able to sense thrombin generated from coagulation. To test this hypothesis, we performed a series of experiments and first confirmed that thrombin triggers TF expression on monocytes from healthy donors (fig. S7A). Subsequently, we found that this effect was associated with reduced expression of PAR-1 on the surface of these cells upon thrombin stimulation (fig. S7B). We observed that TF expression triggered by thrombin was restricted to $\mathrm{CD} 14^{\text {high }}$ monocytes (fig. S7C), and we further established that $\mathrm{CD} 14^{++} \mathrm{CD} 16^{-}$and $\mathrm{CD} 14^{+} \mathrm{CD} 16^{+}$cells exhibited similarly high expression of PAR-1 (fig. S7C). Additional assays in $\mathrm{HIV}^{+}$patients suggested that PAR-1 is preferentially expressed in $\mathrm{CD} 14^{\text {high }}$ cells and that it is shed in response to thrombin in monocytes, a phenomenon that was associated with induction of TF expression by these cells (fig. S7D). These results indicate that the subset of monocytes that produces TF upon LPS-driven activation is the same that can respond to thrombin stimulation in vitro, possibly via PAR-1. bin also produce multiple proinflammatory cytokines. We tested this idea by examining cytokine production by PBMC directly stimulated with thrombin in vitro. We found that thrombin induced robust production of intracellular cytokines (TNF- $\alpha$, IL- $1 \beta$, and IL-6) by CD14 $4^{\text {high }}$ monocytes from healthy individuals (fig. S7E). Pharmacologic inhibition of PAR-1 signaling by SCH79797 completely neutralized the effects of thrombin on TF expression and cytokine production (fig. S7E). These results delineate how thrombin triggers an inflammatory response by monocytes in a PAR-1-dependent manner.

\section{Ixolaris blocks TF activity without suppressing monocyte immune function in vitro}

The results at this point support the hypothesis that monocytes are highly responsive to thrombin and act as a critical link between TLRdriven persistent inflammation and coagulation in chronic viral infections. Interventional therapies focusing on TF inhibition and/or blockade could therefore be key to breaking this vicious cycle of coagulation and inflammation in HIV and SIV pathogenesis. Ixolaris is a small molecule isolated from the saliva of the tick Ixodes scapularis and acts as a potent 
TF pathway inhibitor, by blocking factor VIIa/TF-induced coagulation $(38,39)$. Although Ixolaris has been tested in thrombosis models $(40)$, it has not been evaluated in the setting of HIV-driven coagulopathy.

In our in vitro model, Ixolaris inhibited TF functional activity in monocytes from healthy donors stimulated with LPS in a dose-dependent fashion (Fig. 5A). Even at doses 10 times higher than the maximum inhibitory concentration $(10 \mathrm{ng} / \mathrm{ml})$, Ixolaris did not exhibit substantial cytotoxicity (fig. S8). We next examined whether Ixolaris blocks TF activity in pathological settings such as HIV and SIV infection. Ixolaris completely inhibited the formation of factor Xa under unstimulated conditions and after LPS stimulation in vitro in PBMC from both ART-naïve and ART-treated, virologically suppressed $\mathrm{HIV}^{+}$persons (Fig. 5B). Ixolaris also significantly inhibited TF activity in PBMC from chronically SIVsab-infected PTMs and AGMs (Fig. 5C). Ixolaris did not affect TF protein expression or cytokine production triggered by LPS stimulation in vitro (Fig. 5D). These results demonstrate that, at low doses, Ixolaris can potently inhibit TF functional activity in cells from $\mathrm{HIV}^{+}$patients and from SIV sab-infected NHPs and suggest that inhibition of TF activity could be used to suppress monocyte-driven activation of coagulation in these settings without directly affecting the capacity of these cells to respond to TLR stimulation.

\section{In vivo administration of Ixolaris is safe and decreases coagulation and immune activation in SIVsab infection in PTMs}

To assess the efficacy of Ixolaris, we first measured its anticoagulant effect in vitro (Fig. 6). Ixolaris resulted in dose-dependent prolongation of prothrombin time (PT)/international normalized ratio (INR) when added to human plasma collected from healthy individuals (Fig. 6A). Changes in PT/INR were similar when Ixolaris was added to plasma from uninfected healthy PTMs (Fig. 6B). Activated partial thromboplastin time (aPTT), an intrinsic pathway coagulation marker, was not affected by Ixolaris in either monkey or human plasma, indicating that Ixolaris has a specific inhibitory effect on the extrinsic pathway. Our results thus confirmed the high efficacy and specificity of Ixolaris in NHPs in vitro.

Chronic progressive HIV/SIV infection is characterized by high levels of immune activation, inflammation, and hypercoagulation, which are robust independent prognostic factors of progression to AIDS and comorbidities $(23,41,42)$. To investigate the effect of the anticoagulant therapy on the outcome of a highly pathogenic SIVsab infection, we administered Ixolaris to five PTMs upon SIVsab infection (at the day of infection) and compared the natural history of SIVsab infection in Ixolaris-treated PTMs and untreated controls. Therefore, we first assessed the effects of the anticoagulant treatment by comparing the levels of immune activation and inflammation markers in PTMs receiving Ixolaris and in controls. The animals treated with Ixolaris showed significantly reduced levels of the proinflammatory cytokine IL-17 during early chronic infection $(P=0.03$; Fig. $6 \mathrm{C})$. The anticoagulant treatment also affected $\mathrm{T}$ cell immune activation, as demonstrated by a lower frequency of $\mathrm{CD} 4^{+} \mathrm{T}$ cells expressing HLA-DR ${ }^{+}$ and $\mathrm{CD} 38^{+}$(Fig. 6D), and by significantly lower frequency of $\mathrm{CD} 8^{+}$ T cells expressing HLA-DR ${ }^{+}$and $\mathrm{CD} 38^{+}$during early chronic infection $(P<0.001$; Fig. 6E).

After treatment, TF expression on circulating $\mathrm{CD} 14^{+}$monocytes decreased compared with untreated controls (Fig. 6F). In addition, Ixolaris treatment significantly lowered both CD80 expression in chronically infected PTMs $(P=0.004$; Fig. $6 \mathrm{G})$ and CD86 expression in both acutely and chronically infected PTMs $(P=0.03$; Fig. $6 \mathrm{H})$ compared with the untreated controls. To further validate the reduction of monocyte activation induced by Ixolaris treatment, we measured glucose transporter-1 (Glut-1) expression on $\mathrm{CD}_{14}{ }^{+}$monocytes, an important monocyte activation marker (43-45). Ixolaris-treated animals showed a significantly reduced Glut- 1 expression after SIV infection
A

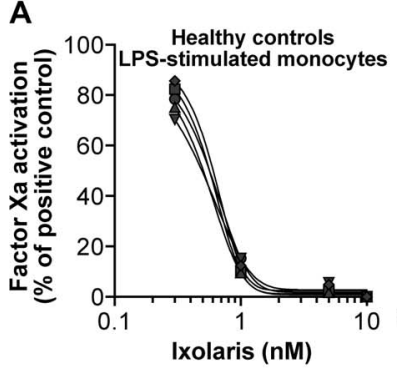

D
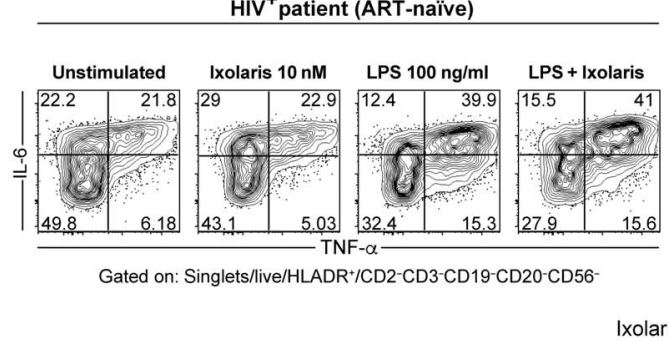

B

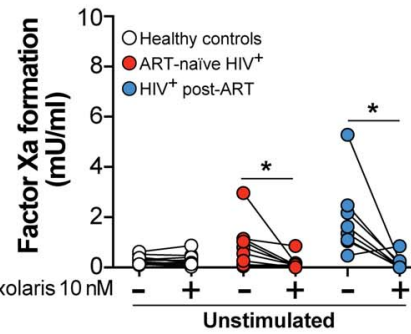

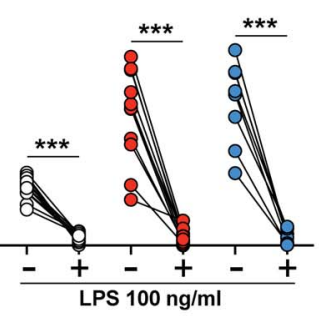

ART-naïve HIV ${ }^{+}$patient - LPS-stimulated PBMC

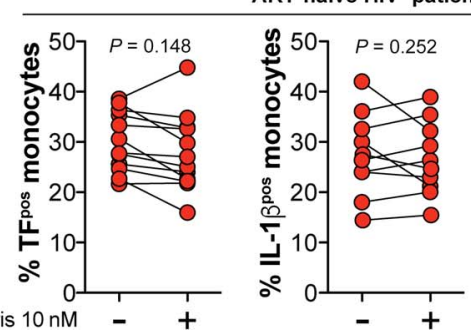

C

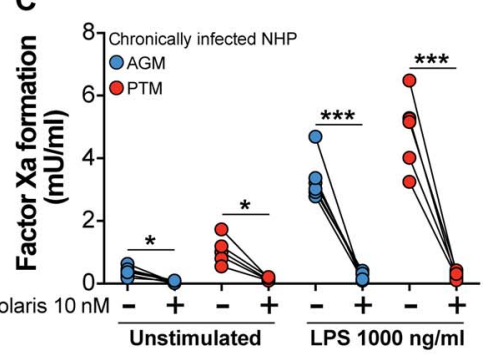

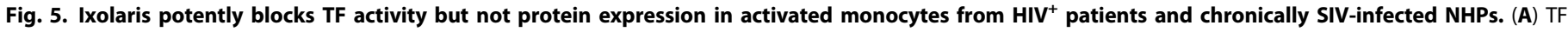

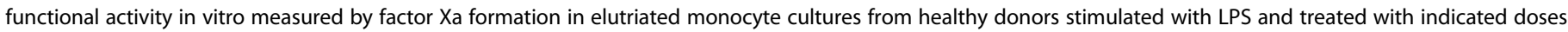

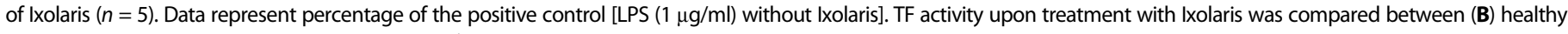

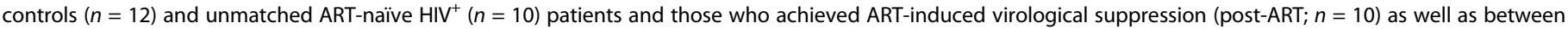

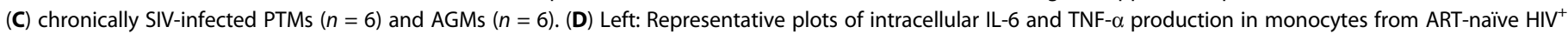

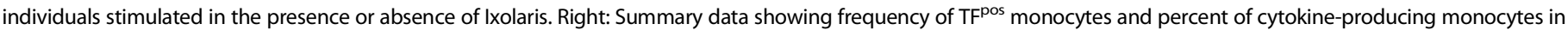
stimulated cultures treated or not with Ixolaris. Data were analyzed using the Wilcoxon matched pairs test. ${ }^{*} P<0.05,{ }^{* * *} P<0.001$. 
A
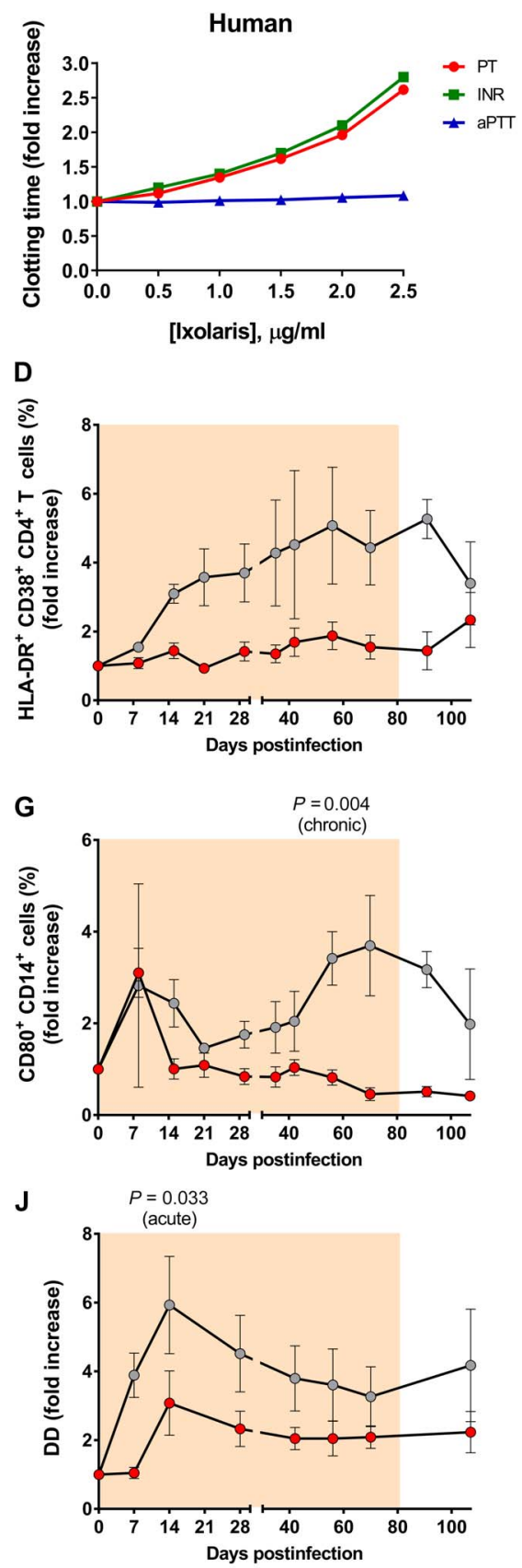

B

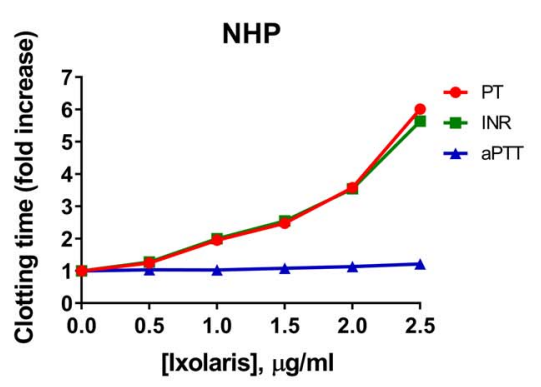

E
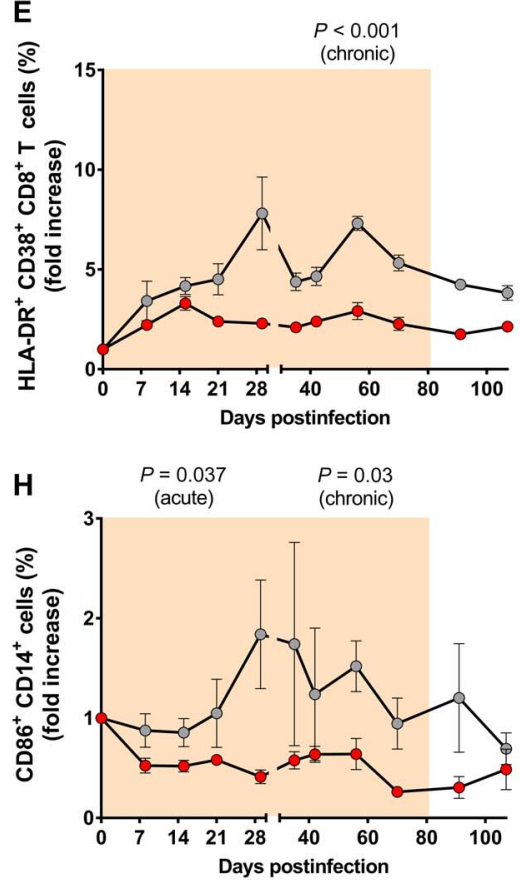

K

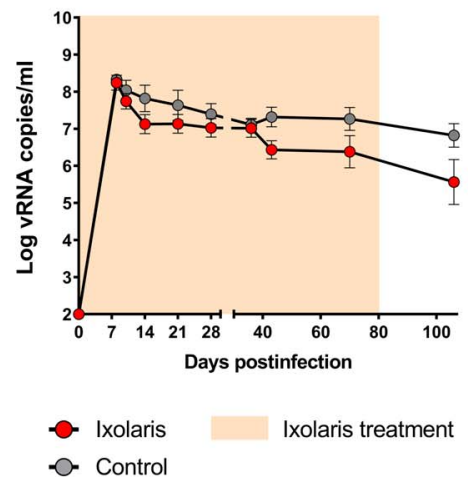

C

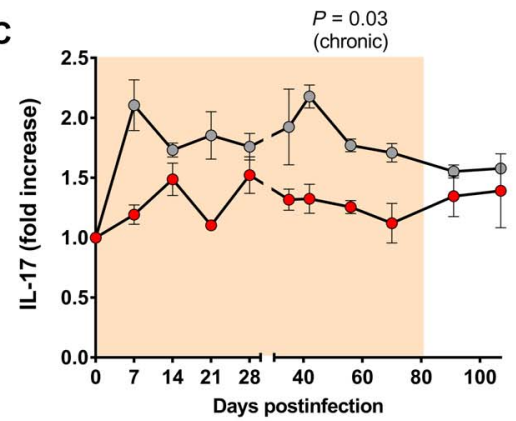

$\mathbf{F}$

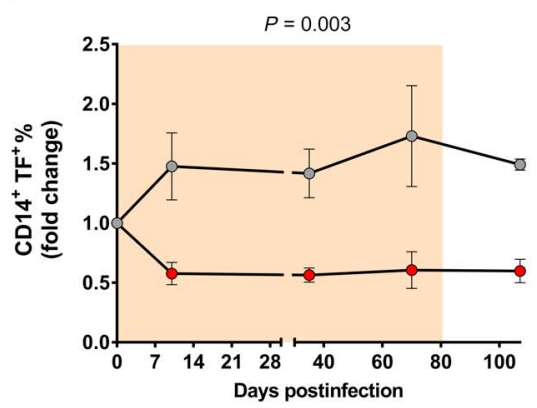

1

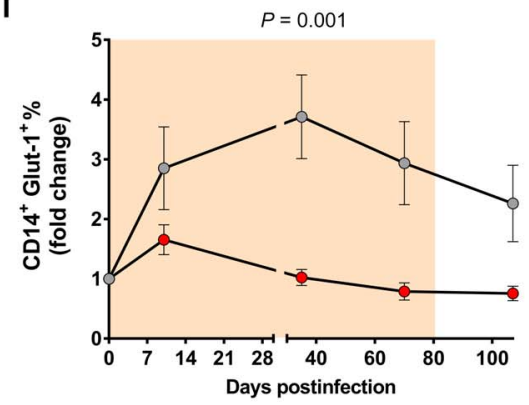

L

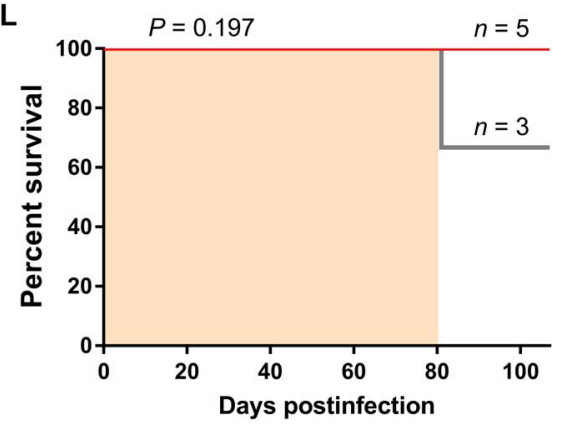

Fig. 6. Anticoagulant treatment positively affects the immune activation and systemic inflammation of highly pathogenic SIVsab infection in PTMs. (A) Dosedependent dynamics of PT/INR and aPTT after Ixolaris addition to plasma isolated from uninfected human subjects. (B) Dose-dependent dynamics of PT/INR and aPTT after Ixolaris addition to plasma isolated from uninfected PTMs. (C) Dynamics of the proinflammatory cytokine IL-17 in untreated versus Ixolaris-treated, SIVsab-infected

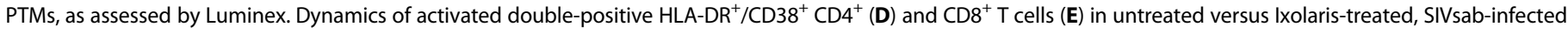
PTMs, as assessed by flow cytometry. Dynamics of TF expression (F) on CD14 $4^{+}$monocytes in untreated versus Ixolaris-treated, SIVsab-infected PTMs, as assessed by flow cytometry, as well as CD80 (G), CD86 (H), and Glut-1 (I) expression on CD14 $4^{+}$monocytes in untreated versus Ixolaris-treated, SIVsab-infected PTMs, as assessed by flow cytometry. (J) Dynamics of D-dimer (DD) in untreated versus Ixolaris-treated, SIVsab-infected PTMs, as assessed by immunoturbidimetric assay. (K) Dynamics of plasma SIVsab viremia in untreated versus Ixolaris-treated, SIVsab-infected PTMs, as assessed by a real-time PCR assay. vRNA, viral RNA. (L) Survival in untreated versus Ixolaristreated, SIVsab-infected PTMs. Statistical analyses were performed with grouping of acute versus chronic infection time points, as described in Materials and Methods, except for TF and Glut-1 for which the statistical analyses were carried out on whole dynamics, due to limited availability of samples for flow staining. Survival analysis was performed using the Mantel-Cox test. 
compared to untreated controls $(P=0.001$; Fig. 6I). Furthermore, we examined associations between expression of monocyte markers and lymphocyte activation using generalizing estimating equations, due to the nature of the data distribution and small sample size of the experimental groups. Using this approach, we found that the monocyte activation markers strongly associated with lymphocyte activation. $\mathrm{CD} 80$ and CD86 expression on $\mathrm{CD} 14^{+}$monocytes were directly associated with HLA-DR ${ }^{+} \mathrm{CD} 38^{+}$expression on $\mathrm{CD} 4^{+} \mathrm{T}$ cells $(P<0.001$ and $P<0.001$, respectively), whereas Glut- 1 expression on $\mathrm{CD} 14^{+}$ monocytes was strongly associated with $\mathrm{HLA}-\mathrm{DR}^{+} \mathrm{CD} 38^{+}$expression on both $\mathrm{CD}^{+}$and $\mathrm{CD}^{+}$T cells $(P<0.001$ and $P<0.001$, respectively). Together, these results suggest that Ixolaris treatment had a beneficial effect in reducing immune activation and inflammation associated with SIV infection.

The hypercoagulable status exhibited in SIVsab-infected progressive NHPs and HIV-infected patients is marked by elevated D-dimer, which is associated with increased incidence of cardiovascular comorbidities and mortality $(21,25,46)$. Therefore, we next compared the D-dimer levels in Ixolaris-treated PTMs and controls. Ixolaris administration significantly reduced plasma D-dimer levels in acute SIV sab infection of PTMs $(P=0.033)$ (Fig. 6J) and resulted in lower D-dimer levels during early chronic infection, indicating a clear effect of Ixolaris in improving the coagulation status in the SIV-infected animals. To monitor the infection in SIVsab-infected PTMs, we measured plasma SIV viremia in all infected animals (47). The Ixolaris-treated group showed overall lower viral loads compared to controls (Fig. 6K).

Ixolaris-treated animals did not develop disease during the first 100 days after infection (Fig. 6L). In accordance with our previous studies in which two of five SIVsab-infected PTMs were rapid progressors $(48,49)$, one of three animals progressed to AIDS in the first 100 days after infection in the untreated group; however, no rapid progression was registered in the Ixolaris group. Statistical significance was not reached in the present study, possibly due to the small sample size. This result is of potential translational interest because it suggests that Ixolaris treatment may abrogate rapid progression in treated animals, likely due to the combined effect of reduced immune activation and inflammation, reduced hypercoagulable status, and small reduction in plasma viremia.

\section{DISCUSSION}

Chronic HIV infection has been associated with elevated circulating levels of biomarkers of coagulation, in particular D-dimer, and systemic inflammation, such as IL-6, sCD14, and CRP $(22,23,50)$, which have been independently linked to a higher risk of non-AIDS-related death and mortality (23) even in persons treated with ART (51). Although ART-induced suppression of HIV viremia is shown to significantly reduce plasma levels of $\mathrm{D}$-dimer, plasma levels remain higher than in non-HIV-infected populations (32).

Monocytes have been previously described as immune cells involved in cardiovascular disease in both $\mathrm{HIV}^{-}$and $\mathrm{HIV}^{+}$populations $(4,51-53)$ and are an important source of TF (9). Here, we performed a detailed immune profiling of monocyte subsets and delineated molecular signatures that characterize TF-expressing cells in the context of TLR activation. Our results demonstrated that $\mathrm{TF}^{\mathrm{pos}}$ monocytes exhibit elevated expression of several genes associated with innate immune activation. These findings argue that the subpopulation of monocytes that is able to up-regulate TF expression upon TLR4 activation is the classical subset defined as $\mathrm{CD} 14^{+} \mathrm{CD} 16^{-}$. Activated monocytes that do not express TF under the same circumstances exhibit markers of patrolling monocytes $\left(\mathrm{CD} 14^{\mathrm{dim}} \mathrm{CD} 16^{+}\right)$such as up-regulation of CX3CR1 gene expression. In the setting of coagulopathy, it is possible that patrolling monocytes, expressing high levels of CX3CR1, a vascular homing receptor, might interact with thrombin clots. Our results indicate that the patrolling monocytes express the lowest level of PAR-1 and that PAR-1 expression is decreased by thrombin stimulation. Therefore, in vivo, it is possible that the interaction between CX3CR1-expressing patrolling monocytes and thrombin clots accounts for the lower expression of PAR-1. Furthermore, we demonstrated that the frequency of monocytes expressing TF upon activation could not be further increased with augmenting doses of TLR activation, suggesting an inherent capacity of these circulating monocytes to express this coagulation factor. We confirmed that nonmonocytic cells from peripheral blood are unable to express TF upon LPS stimulation. Flow cytometry-based phenotypic analysis revealed that compared to $\mathrm{TF}^{\text {neg }}$ monocytes, $\mathrm{TF}^{\mathrm{pos}}$ cells displayed increased expression of HLA-DR and costimulatory molecules such as CD40 and CD86 as well as IL-6R. These results indicate a unique monocyte phenotype capable of producing TF. Previous flow cytometry-based studies have indicated that $\mathrm{CD} 16^{+}$monocytes are able to produce TF (54). However, our results indicate that the molecular signature associated with TF expression in monocytes is restricted to the classical proinflammatory subset that lacks expression of CX3CR1. It is possible that different experimental settings and/or gating strategies have resulted in discrepancies between the studies, and for this reason, we performed TF expression assessment in sorted monocyte subsets to identify the main TF producers. In addition, molecular gene analysis was performed in healthy individuals, and gene expression might differ in $\mathrm{HIV}^{+}$individuals due to the trained immunity phenomenon (55). Our findings indicate that classical proinflammatory monocytes are the major subset capable of robustly expressing TF protein and also up-regulating TF functional activity upon LPS stimulation.

Previous studies have indicated that TF expression on monocytes is increased in HIV-infected individuals compared to healthy controls and that the frequency of $\mathrm{TF}^{\text {pos }}$ cells is associated with HIV disease progression (18). Here, we found that the frequency of $\mathrm{TF}^{\mathrm{pos}}$ monocytes, as well as TF functional activity, was similar between ART-naïve $\mathrm{HIV}^{+}$individuals and those who achieved HIV suppression after ART initiation. These findings strongly indicate that TF expression by monocytes remains high in $\mathrm{HIV}^{+}$individuals regardless of ART-induced virological suppression, which could be associated with increased potential to activate the coagulation cascade and cause cardiovascular disease. Consistent with this concept, previous work has demonstrated a positive correlation between frequency of $\mathrm{TF}^{\mathrm{pos}}$ monocytes and D-dimer levels in $\mathrm{HIV}^{+}$patients (18). We validated these findings in our analysis of ART-naive $\mathrm{HIV}^{+}$patients. Intriguingly, although the D-dimer plasma levels were significantly reduced after ART-induced suppression of HIV viremia, the frequency of $\mathrm{TF}^{\mathrm{pos}}$ monocytes remained elevated. It is plausible that some degree of coagulopathy persists despite the decreases in D-dimer and supports a more complex relationship between pro- and anticoagulant factors in untreated HIV due to poor synthetic liver function (20). This also suggests that assessing TF expression on monocytes would increase sensitivity for the detection of coagulopathy. Our findings further reveal TF as a potential therapeutic target in ART-treated patients with evidence of coagulopathy.

The pathogenic role of coagulation during progressive SIV sab infection in PTMs has been established previously (25), and NHPs serve as an important HIV model that can be controlled for key variables, such as time of infection. Here, we observed that compared to AGMs, which experience active viral replication but no disease progression, PTMs 
have significantly increased frequency of TF-expressing monocytes and TF functional activity upon chronic SIV sab infection. No differences were observed in TF expression and activity in vitro between PTMs and AGMs before SIV infection, suggesting that monocyte subsets from these two NHP species diverge markedly in response to chronic viral infection. These results also link the occurrence of SIV progression and systemic coagulopathy with increased capacity of monocytes to produce TF upon activation.

Aside from their role in coagulation, monocytes are also important in inflammatory processes due to their production of cytokines. There is a growing body of evidence indicating that persistent inflammation is associated with increased mortality in $\operatorname{HIV}(23,56)$. Our experiments assessing polyfunctionality of monocytes by means of production of IL- $1 \beta$, IL-6, and TNF- $\alpha$ upon TLR activation in vitro demonstrate that $\mathrm{TF}^{\mathrm{pos}}$ cells are more frequently triple cytokine producers when compared to $\mathrm{TF}^{\text {neg }}$ monocytes in healthy individuals and in $\mathrm{HIV}^{+}$patients, as well as in chronically SIV-infected PTMs. These results suggest that the same monocyte subset that expresses TF upon activation may also be implicated in persistent inflammation by producing multiple cytokines. This idea was reinforced by our findings demonstrating an increased frequency of polyfunctional monocytes in PTMs compared to AGMs after SIVsab infection but not in naïve animals. Thus, it is reasonable to propose that $\mathrm{TF}^{\text {pos }}$ cells may be critically implicated in the promotion of systemic inflammation and coagulation associated with disease progression in chronic HIV and SIVsab infection (fig. S9).

Having identified a role of TF-expressing monocytes at the intersection of inflammation and coagulation, we hypothesized that interfering with TF could serve as a therapeutic approach to target hypercoagulation. Administration of Ixolaris in vivo decreased $\mathrm{T}$ cell activation as well as plasma IL-17 and D-dimer levels. Ixolaris produced no evidence of toxicity and was not associated with significant $\mathrm{CD} 4^{+}$or SIVsab viremia changes. The results of the in vivo study are significant for two reasons. First, they point to a causal relationship between coagulation and immune activation and inflammation. Thus, the Ixolaris treatment did not only reduce inflammation but also lowered monocyte and lymphocyte activation in the treated animals. The reduction of lymphocyte activation may be the direct result of reduced expression of the costimulatory markers CD80 and CD86 (two potent T cell activators) on monocytes. Alternatively, Ixolaris treatment could have affected the levels of immune activation by directly reducing $\mathrm{T}$ cell expression of PAR-1. Such a scenario is supported by our finding that Ixolaris treatment appears to have a stronger impact on the activation of $\mathrm{CD}^{+} \mathrm{T}$ cell activation because these cells express higher levels of PAR-1. In vitro assays showed that Ixolaris treatment did not diminish TF expression on monocytes in response to LPS; however, these assays did not have the capacity to generate thrombin. The in vivo data show reduction of monocyte activation after Ixolaris treatment, highlighting the importance of TF-generated thrombin on monocyte activation.

Our study limitations included the small sample size of our longitudinal cohort and the cross-sectional nature of many experiments. In addition, the in vivo Ixolaris administration study included only a small number of ART-naïve acutely infected animals, prohibiting a more detailed evaluation of possible toxicity, drug interaction, complications, and potential survival benefit in untreated or treated animals.

The impact of anticoagulant therapy on immune activation and inflammation in SIVsab-infected PTMs demonstrates that hypercoagulation is a significant source of persistent immune activation and inflammation in this model and probably in HIV-infected patients as well. Anticoagulant therapy by itself reduced important measurements of immune activation and inflammation and therefore has potential to improve the clinical management of HIV-infected patients. Second, we show that anticoagulant treatment improved the natural history of highly pathogenic SIVsab infection even in the absence of any other intervention aimed at either controlling viral replication (ART) or improving the health of the gut. Therefore, our study suggests that targeting the coagulation pathway in HIV-infected patients may be effective in reducing the immune activation and inflammation that are linked to cardiovascular comorbidities in HIV infection. These findings indicate that targeting TF may be used as a host-directed therapy in chronic HIV infection as well as other inflammatory diseases with similar immunopathology.

\section{MATERIALS AND METHODS Study design}

Ethics statements are detailed in the Supplementary Materials and Methods. Participants were prospectively enrolled in a clinical observational study to evaluate the impact of ART in ART-naïve HIV-infected persons. The study objectives included the evaluation of phenotype and function of monocytes at different stages of HIV infection. Participants were selected retrospectively from a completed trial for evaluation of the monocyte function based on PBMC availability, compliance with ART, and lack of aberrant immune responses or clinical course that would entail administration of medications that may affect coagulation such as corticosteroids or chemotherapy or other immune therapies.

For the in vivo NHP studies, eight PTMs were infected with SIVsab. In PTMs, Ixolaris was administered starting from the day of the infection to assess its impact on key parameters of SIV infection (survival, viral replication, $\mathrm{CD} 4^{+} \mathrm{T}$ cell counts and activation status, systemic inflammation, and coagulation status). The study design involved the assessment of the impact of Ixolaris on key variables of acute SIV infection because this stage of infection is when the differences in these parameters can reach statistical significance even in small animal groups. In addition, the study design permitted the comparisons not only between the animals in the Ixolaris-treated group and controls but also in the same animals before and after SIVsab infection and Ixolaris treatment. Multiple samples collected at well-defined key time points of SIVsab infection enabled these comparisons. Primary data are located in table S6.

\section{Description of HIV-infected patients}

Cross-sectional analysis was performed in HIV-infected individuals enrolled in protocols at the National Institutes of Health. Cryopreserved PBMC from $10 \mathrm{HIV}^{+}$ART-naïve donors and $10 \mathrm{HIV}^{+}$virally suppressed patients (median, 128 weeks on ART; IQR, 112 to 128 weeks) were used. The characteristics of the participants from the cross-sectional analysis are shown in table S2. Prospective analysis was performed in HIVinfected ART-naïve patients over the age of 18 with $\mathrm{CD} 4^{+} \mathrm{T}$ cell counts of $<100$ cells/ $\mu$ l before therapy initiation. Cryopreserved PBMC samples were used from 12 patients pre- and post-ART initiation after HIV suppression (median, 160 weeks on ART; IQR, 96 to 192 weeks). The characteristics of the participants from the prospective analysis are shown in table S3. Blood from age- and gender-matched healthy donors was collected from the National Institutes of Health blood bank.

\section{Description of the NHPs samples used for in vitro and in vivo studies}

Cryopreserved PBMC from six PTMs and six AGMs were studied from pre-SIV sab infection $\left(\mathrm{SIV}^{-}\right.$, naïve) and 72 days post-SIVsab infection 
$\left(\mathrm{SIV}^{+}\right)$. The characteristics of the animals before and after SIVsab infection are shown in table S1. For the preclinical study in vivo, eight PTMs were intravenously infected with plasma equivalent to 300 tissue culture infectious doses (TCID50) of SIV sab strain BH66 (48). Ixolaris therapy (20 $\mu \mathrm{g} / \mathrm{kg}$, subcutaneously, daily) was initiated in five PTMs at the time of infection and was maintained for 80 days. Blood was collected from all animals twice before infection, twice a week for the first 2 weeks postinfection, weekly during early chronic infection (up to 10 weeks postinfection), and then monthly. The Ixolaris-treated group was closely monitored for signs of bleeding. Plasma viral RNA loads were quantified using real-time qPCR specifically developed for SIVsab, as described previously $(57,58)$.

\section{LPS stimulation}

Cryopreserved PBMC were thawed and resuspended in RPMI 1640 supplemented with $10 \%$ human $\mathrm{AB}$ serum at $10^{6}$ cells per well in 96-well plates. Cells were washed and resuspended in complete media with brefeldin-A ( $5 \mu \mathrm{g} / \mathrm{ml}$; Sigma-Aldrich) and stimulated with indicated doses of ultrapure LPS (Sigma-Aldrich) in the presence or absence of purified Ixolaris $(10 \mathrm{nM})$ for 6 hours at $37^{\circ} \mathrm{C}$ in $5 \% \mathrm{CO}_{2}$. Cells were then stained following the flow cytometry assay described above.

\section{In vitro experiments with $\mathrm{HIV}^{+}$and $\mathrm{HCV}^{+}$serum}

Column-purified $\mathrm{CD} 14^{+}$monocytes were obtained from healthy blood donors and resuspended in RPMI 1640 supplemented with $10 \%$ of indicated serum (commercialized human $\mathrm{AB}$ serum or pooled $0.22-\mu \mathrm{m}$ filtered sera from 12 healthy controls, $20 \mathrm{ART}$-naïve $\mathrm{HIV}^{+}$patients and at week 12 and 48 of ART, and $16 \mathrm{HCV}^{+}$persons) at $10^{6}$ cells per well in 96 -well plates. Cells were left overnight (18 hours) at $37^{\circ} \mathrm{C}$ in 5\% $\mathrm{CO}_{2}$. Cells were then washed and lysed for measurement of $\mathrm{TF}$ protein expression in cell lysates. In additional experiments, cells were incubated with anti-IL1R1 (10 $\mu \mathrm{g} / \mathrm{ml}$; polyclonal, R\&D Systems), antiIL6Ra $(10 \mu \mathrm{g} / \mathrm{ml}$; clone 17506, R\&D Systems), anti-IFN $\gamma \mathrm{R}(10 \mu \mathrm{g} / \mathrm{ml}$; clone $92101, \mathrm{R} \& \mathrm{D}$ Systems), anti-IFNAR $(10 \mu \mathrm{g} / \mathrm{ml}$; polyclonal, ab10719, Abcam), and anti-TNFR1 (10 $\mu \mathrm{g} / \mathrm{ml}$; clone 16803R, R\&D Systems) for 1 hour before incubation with $\mathrm{HIV}^{+}$serum and persisted in cultures for additional 18 hours. $\mathrm{HIV}^{+}$serum was also treated with polymyxin $(0.5 \mu \mathrm{g} / \mu \mathrm{l}$; Sigma-Aldrich) for 1 hour and filtered before incubating for 18 hours.

\section{Testing the efficacy of Ixolaris in inhibiting coagulation in NHP in vitro}

The in vitro efficacy of Ixolaris was assessed by measuring its impact on clotting time on citrate plasma from SIV-naïve healthy PTMs. Results were compared to the in vitro testing of Ixolaris-treated plasma from healthy human subjects. Plasma samples from PTMs and humans were incubated with Ixolaris $(0.5,1,1.5,2$, or $2.5 \mu \mathrm{g} / \mathrm{ml})$ for $2 \mathrm{~min}$ at $37^{\circ} \mathrm{C}$. PT/INR and aPTT were then immediately measured. Testing was performed by ITxM Diagnotics.

\section{Statistical analysis}

Median and interquartile ranges were used as measures of central tendency. All statistical comparisons were prespecified and two-tailed. Differences with $P<0.05$ were considered statistically significant. Data from continuous variables comparing two groups were analyzed using Wilcoxon matched-pairs, Mann-Whitney, and Spearman rank correlation tests. Comparisons between more than two groups were performed using the Kruskal-Wallis test with Dunn's multiple comparisons ad hoc test. Nominal variables and the expression profile of cytokine produc- tion by monocytes were compared using the Fisher's exact test (two groups) or $\chi^{2}$ test (more than two groups). Hierarchical cluster analysis (Ward's method with bootstrap 100x) was used to test whether TF ${ }^{\text {pos }}$ and $\mathrm{TF}^{\text {neg }}$ monocytes could be clustered separately on the basis of expression profile of selected proinflammatory genes measured by $\mathrm{qPCR}$ (after data was $z$-score-normalized). A model of PCA with an associated vector analysis was used to visualize the distinction between $\mathrm{TF}^{\mathrm{pos}}$ and $\mathrm{TF}^{\text {neg }}$ cells and the direction in which each marker influences mathematically the distribution of the data. These data analyses were performed using GraphPad Prism 6.0 (GraphPad Software Inc.) and JMP 11.0 (SAS) softwares. Additional description of statistical analyses is available in the Supplementary Materials and Methods.

\section{SUPPLEMENTARY MATERIALS}

www.sciencetranslationalmedicine.org/cgi/content/full/9/405/eaam5441/DC1 Materials and Methods

Fig. S1. Gating strategy used to evaluate monocytes in PBMC

Fig. S2. Detailed phenotyping of TF-expressing monocytes.

Fig. S3. Frequency of TF-expressing monocytes in patients prospectively undergoing ART and its relationship with CRP and D-dimer.

Fig. S4. Circulating CD14 ${ }^{+}$monocytes, but not myeloid dendritic cells, express TF in chronically SIV-infected PTMs.

Fig. S5. Induction of TF expression in the gut of PTMs infected with SIVab.

Fig. S6. Intracellular cytokine production and TF expression in HIV-infected patients and in SIVab-infected NHPs.

Fig. S7. Thrombin induces TF expression on CD14 ${ }^{\text {high }}$ monocytes via PAR-1.

Fig. S8. Cell viability upon treatment with Ixolaris and/or LPS in vitro.

Fig. S9. Activated monocytes expressing TF represent a link between coagulation and inflammation.

Table S1. Characteristics of the NHPs used for the in vitro studies.

Table S2. Characteristics of HIV-infected individuals included in the cross-sectional analysis. Table S3. Characteristics of HIV-infected individuals included in the prospective analyses. Table S4. List of antibodies used in the flow cytometry experiments in both human and NHP samples.

Table S5. List of human primers.

Table S6. Primary data.

References (59-62)

\section{REFERENCES AND NOTES}

1. G. Lauvau, L. Chorro, E. Spaulding, S. M. Soudja, Inflammatory monocyte effector mechanisms. Cell. Immunol. 291, 32-40 (2014).

2. F. Ginhoux, S. Jung, Monocytes and macrophages: Developmental pathways and tissue homeostasis. Nat. Rev. Immunol. 14, 392-404 (2014).

3. N. G. Sandler, H. Wand, A. Roque, M. Law, M. C. Nason, D. E. Nixon, C. Pedersen, K. Ruxrungtham, S. R. Lewin, S. Emery, J. D. Neaton, J. M. Brenchley, S. G. Deeks, I. Sereti, D. C. Douek; Insight Smart Study Group, Plasma levels of soluble CD14 independently predict mortality in HIV infection. J. Infect. Dis. 203, 780-790 (2011).

4. J. V. Baker, K. H. Hullsiek, A. Singh, E. Wilson, K. Henry, K. Lichtenstein, N. Onen, E. Kojic, P. Patel, J. T. Brooks, H. N. Hodis, M. Budoff, I. Sereti; CDC SUN Study Investigators, Immunologic predictors of coronary artery calcium progression in a contemporary HIV cohort. AIDS 28, 831-840 (2014).

5. E. M. P. Wilson, A. Singh, K. H. Hullsiek, D. Gibson, W. K. Henry, K. Lichtenstein, N. F. Önen, E. Kojic, P. Patel, J. T. Brooks, I. Sereti, J. V. Baker; Natural History of HIV/AIDS in the Era of Effective Therapy (SUN Study) Investigators, Monocyte-activation phenotypes are associated with biomarkers of inflammation and coagulation in chronic HIV infection. J. Infect. Dis. 210, 1396-1406 (2014).

6. R. A. McKibben, J. B. Margolick, S. Grinspoon, X. Li, F. J. Palella Jr., L. A. Kingsley, M. D. Witt, R. T. George, L. P. Jacobson, M. Budoff, R. P. Tracy, T. T. Brown, W. S. Post, Elevated levels of monocyte activation markers are associated with subclinical atherosclerosis in men with and those without HIV infection. J. Infect. Dis. 211, 1219-1228 (2015).

7. B. B. Andrade, A. Singh, G. Narendran, M. E. Schechter, K. Nayak, S. Subramanian, S. Anbalagan, S. M. R. Jensen, B. O. Porter, L. R. Antonelli, K. A. Wilkinson, R. J. Wilkinson, G. Meintjes, H. van der Plas, D. Follmann, D. L. Barber, S. Swaminathan, A. Sher, I. Sereti, Mycobacterial antigen driven activation of $\mathrm{CD} 14^{++} \mathrm{CD} 16^{-}$monocytes is a predictor of tuberculosis-associated immune reconstitution inflammatory syndrome. PLOS Pathog. 10, e1004433 (2014). 
8. G. J. Broze Jr., Binding of human factor VII and VIla to monocytes. J. Clin. Invest. 70, 526-535 (1982).

9. B. Osterud, E. Bjorklid, Tissue factor in blood cells and endothelial cells. Front. Biosci. (Elite Ed.) 4, 289-299 (2012).

10. W. Ruf, Protease-activated receptor signaling in the regulation of inflammation. Crit. Care Med. 32, S287-S292 (2004).

11. H. Kothari, L. V. M. Rao, R. Vankayalapati, U. R. Pendurthi, Mycobacterium tuberculosis infection and tissue factor expression in macrophages. PLOS ONE 7, e45700 (2012).

12. B. P. Tsao, D. S. Fair, L. K. Curtiss, T. S. Edgington, Monocytes can be induced by lipopolysaccharide-triggered T lymphocytes to express functional factor VII/VIla protease activity. J. Exp. Med. 159, 1042-1057 (1984).

13. S. M. Opal, C. T. Esmon, Bench-to-bedside review: Functional relationships between coagulation and the innate immune response and their respective roles in the pathogenesis of sepsis. Crit. Care 7, 23-38 (2002).

14. C. T. Esmon, Does inflammation contribute to thrombotic events? Haemostasis $\mathbf{3 0}$ (suppl. 2), 34-40 (2000)

15. M. Levi, T. van der Poll, H. R. Büller, Bidirectional relation between inflammation and coagulation. Circulation 109, 2698-2704 (2004).

16. A. P. Owens III, N. Mackman, Role of tissue factor in atherothrombosis. Curr. Atheroscler. Rep. 14, 394-401 (2012).

17. H. Pawelski, D. Lang, S. Reuter, Interactions of monocytes and platelets: Implication for life. Front. Biosci. (Schol. Ed.) 6, 75-91 (2014).

18. N. T. Funderburg, E. Mayne, S. F. Sieg, R. Asaad, W. Jiang, M. Kalinowska, A. A. Luciano, W. Stevens, B. Rodriguez, J. M. Brenchley, D. C. Douek, M. M. Lederman, Increased tissue factor expression on circulating monocytes in chronic HIV infection: Relationship to in vivo coagulation and immune activation. Blood 115, 161-167 (2010).

19. N. T. Funderburg, M. M. Lederman, Coagulation and morbidity in treated HIV infection. Thromb. Res. 133 (suppl. 1), S21-S24 (2014).

20. J. V. Baker, K. Brummel-Ziedins, J. Neuhaus, D. Duprez, N. Cummins, D. Dalmau, J. DeHovitz, C. Lehmann, A. Sullivan, I. Woolley, L. Kuller, J. D. Neaton, R. P. Tracy; INSIGHT SMART Study Team, HIV replication alters the composition of extrinsic pathway coagulation factors and increases thrombin generation. J. Am. Heart Assoc. 2, e000264 (2013).

21. D. R. Boulware, K. H. Hullsiek, C. E. Puronen, A. Rupert, J. V. Baker, M. A. French, P. R. Bohjanen, R. M. Novak, J. D. Neaton, I. Sereti; INSIGHT Study Group, Higher levels of CRP, D-dimer, IL-6, and hyaluronic acid before initiation of antiretroviral therapy (ART) are associated with increased risk of AIDS or death. J. Infect. Dis. 203, 1637-1646 (2011).

22. D. A. Duprez, J. Neuhaus, L. H. Kuller, R. Tracy, W. Belloso, S. De Wit, F. Drummond, H. C. Lane, B. Ledergerber, J. Lundgren, D. Nixon, N. I. Paton, R. J. Prineas, J. D. Neaton; INSIGHT SMART Study Group, Inflammation, coagulation and cardiovascular disease in HIV-infected individuals. PLOS ONE 7, e44454 (2012).

23. L. H. Kuller, R. Tracy, W. Belloso, S. De Wit, F. Drummond, H. C. Lane, B. Ledergerber, J. Lundgren, J. Neuhaus, D. Nixon, N. I. Paton, J. D. Neaton; INSIGHT SMART Study Group, Inflammatory and coagulation biomarkers and mortality in patients with HIV infection. PLOS Med. 5, e203 (2008).

24. K. P. High, M. Brennan-Ing, D. B. Clifford, M. H. Cohen, J. Currier, S. G. Deeks, S. Deren, R. B. Effros, K. Gebo, J. J. Goronzy, A. C. Justice, A. Landay, J. Levin, P. G. Miotti, R. J. Munk, H. Nass, C. R. Rinaldo Jr., M. G. Shlipak, R. Tracy, V. Valcour, D. E. Vance, J. D. Walston, P. Volberding; OAR Working Group on HIV and Aging, HIV and aging: State of knowledge and areas of critical need for research. A report to the NIH Office of AIDS Research by the HIV and Aging Working Group. J. Acquir. Immune Defic. Syndr. 60 (suppl. 1), S1-S18 (2012).

25. I. Pandrea, E. Cornell, C. Wilson, R. M. Ribeiro, D. Ma, J. Kristoff, C. Xu, G. S. Haret-Richter, A. Trichel, C. Apetrei, A. Landay, R. Tracy, Coagulation biomarkers predict disease progression in SIV-infected nonhuman primates. Blood 120, 1357-1366 (2012).

26. E. Grage-Griebenow, H.-D. Flad, M. Ernst, Heterogeneity of human peripheral blood monocyte subsets. J. Leukoc. Biol. 69, 11-20 (2001).

27. I. M. Francischetti, E. Gordon, B. Bizzarro, N. Gera, B. B. Andrade, F. Oliveira, D. Ma, T. C. F. Assumpcao, J. M. Ribeiro, M. Pena, C.-F. Qi, A. Diouf, S. E. Moretz, C. A. Long, H. C. Ackerman, S. K. Pierce, A. Sá-Nunes, M. Waisberg, Tempol, an intracellular antioxidant, inhibits tissue factor expression, attenuates dendritic cell function, and is partially protective in a murine model of cerebral malaria. PLOS ONE 9, e87140 (2014).

28. N. T. Funderburg, Markers of coagulation and inflammation often remain elevated in ART-treated HIV-infected patients. Curr. Opin. HIV AIDS 9, 80-86 (2014).

29. D. Ma, A. Jasinska, J. Kristoff, J. P. Grobler, T. Turner, Y. Jung, C. Schmitt, K. Raehtz, F. Feyertag, N. Martinez Sosa, V. Wijewardana, D. S. Burke, D. L. Robertson, R. Tracy, I. Pandrea, N. Freimer, C. Apetrei; International Vervet Research Consortium, SIVagm Infection in wild African green monkeys from south Africa: Epidemiology, natural history, and evolutionary considerations. PLOS Pathog. 9, e1003011 (2013).

30. D. Ma, A. J. Jasinska, F. Feyertag, V. Wijewardana, J. Kristoff, T. He, K. Raehtz, C. A. Schmitt, Y. Jung, J. D. Cramer, M. Dione, M. Antonio, R. Tracy, T. Turner, D. L. Robertson, I. Pandrea,
N. Freimer, C. Apetrei, Factors associated with siman immunodeficiency virus transmission in a natural African nonhuman primate host in the wild. J. Virol. 88, 5687-5705 (2014).

31. G. Marchetti, C. Tincati, G. Silvestri, Microbial translocation in the pathogenesis of HIV infection and AIDS. Clin. Microbiol. Rev. 26, 2-18 (2013).

32. J. Neuhaus, D. R. Jacobs Jr., J. V. Baker, A. Calmy, D. Duprez, A. La Rosa, L. H. Kuller, S. L. Pett, M. Ristola, M. J. Ross, M. G. Shlipak, R. Tracy, J. D. Neaton, Markers of inflammation, coagulation, and renal function are elevated in adults with HIV infection. J. Infect. Dis. 201, 1788-1795 (2010).

33. J. Kalz, H. ten Cate, H. M. H. Spronk, Thrombin generation and atherosclerosis. J. Thromb. Thrombolysis 37, 45-55 (2014).

34. V. Shpacovitch, M. Feld, M. D. Hollenberg, T. A. Luger, M. Steinhoff, Role of proteaseactivated receptors in inflammatory responses, innate and adaptive immunity. J. Leukoc. Biol. 83, 1309-1322 (2008).

35. R. Colognato, J. R. Slupsky, M. Jendrach, L. Burysek, T. Syrovets, T. Simmet, Differential expression and regulation of protease-activated receptors in human peripheral monocytes and monocyte-derived antigen-presenting cells. Blood 102, 2645-2652 (2003).

36. M. L. López, G. Bruges, G. Crespo, V. Salazar, P.-A. Deglesne, H. Schneider, H. Cabrera-Fuentes, M. L. Schmitz, K. T. Preissner, Thrombin selectively induces transcription of genes in human monocytes involved in inflammation and wound healing. Thromb. Haemost. 112, 992-1001 (2014).

37. S. R. Coughlin, Protease-activated receptors in hemostasis, thrombosis and vascular biology. J. Thromb. Haemost. 3, 1800-1814 (2005).

38. I. M. Francischetti, J. G. Valenzuela, J. F. Andersen, T. N. Mather, J. M. C. Ribeiro, Ixolaris, a novel recombinant tissue factor pathway inhibitor (TFPI) from the salivary gland of the tick, Ixodes scapularis: Identification of factor $\mathrm{X}$ and factor $\mathrm{Xa}$ as scaffolds for the inhibition of factor Vlla/tissue factor complex. Blood 99, 3602-3612 (2002).

39. T. C. Carneiro-Lobo, F. Schaffner, J. Disse, H. Ostergaard, I. M. Francischetti, R. Q. Monteiro, W. Ruf, The tick-derived inhibitor Ixolaris prevents tissue factor signaling on tumor cells. J. Thromb. Haemost. 10, 1849-1858 (2012).

40. R. A. Nazareth, L. S. Tomaz, S. Ortiz-Costa, G. C. Atella, J. M. C. Ribeiro, I. M. B. Francischetti, R. Q. Monteiro, Antithrombotic properties of Ixolaris, a potent inhibitor of the extrinsic pathway of the coagulation cascade. Thromb. Haemost. 96, 7-13 (2006).

41. J. L. Fahey, Cytokines, plasma immune activation markers, and clinically relevant surrogate markers in human immunodeficiency virus infection. Clin. Diagn. Lab. Immunol. 5, 597-603 (1998).

42. P. W. Hunt, HIV and inflammation: Mechanisms and consequences. Curr. HIV/AIDS Rep. 9 139-147 (2012).

43. T. R. Butterfield, D. B. Hanna, R. C. Kaplan, J. R. Kizer, H. G. Durkin, M. A. Young, M. J. Nowicki, P. C. Tien, E. T. Golub, M. A. Floris-Moore, K. Titanji, M. A. Fischl, S. L. Heath, J. Martinson, S. M. Crowe, C. S. Palmer, A. L. Landay, J. J. Anzinger, Increased glucose transporter-1 expression on intermediate monocytes from HIV-infected women with subclinical cardiovascular disease. AIDS 31, 199-205 (2017).

44. A. J. Freemerman, A. R. Johnson, G. N. Sacks, J. J. Milner, E. L. Kirk, M. A. Troester, A. N. Macintyre, P. Goraksha-Hicks, J. C. Rathmell, L. Makowski, Metabolic reprogramming of macrophages: Glucose transporter 1 (GLUT1)-mediated glucose metabolism drives a proinflammatory phenotype. J. Biol. Chem. 289, 7884-7896 (2014).

45. C. S. Palmer, J. J. Anzinger, J. Zhou, M. Gouillou, A. Landay, A. Jaworowski, J. M. McCune, S. M. Crowe, Glucose transporter 1-expressing proinflammatory monocytes are elevated in combination antiretroviral therapy-treated and untreated $\mathrm{HIV}^{+}$subjects. J. Immunol. 193, 5595-5603 (2014).

46. I. Pandrea, A. Landay, C. Wilson, J. Stock, R. Tracy, C. Apetrei, Using the pathogenic and nonpathogenic nonhuman primate model for studying non-AIDS comorbidities. Curr. HIV/AIDS Rep. 12, 54-67 (2015).

47. I. Pandrea, C. Apetrei, Where the wild things are: Pathogenesis of SIV infection in African nonhuman primate hosts. Curr. HIV/AIDS Rep. 7, 28-36 (2010).

48. D. T. Mandell, J. Kristoff, T. Gaufin, R. Gautam, D. Ma, N. Sandler, G. Haret-Richter, C. Xu, H. Aamer, J. Dufour, A. Trichel, D. C. Douek, B. F. Keele, C. Apetrei, I. Pandrea, Pathogenic features associated with increased virulence upon simian immunodeficiency virus cross-species transmission from natural hosts. J. Virol. 88, 6778-6792 (2014).

49. I. Pandrea, C. Xu, J. L. Stock, D. N. Frank, D. Ma, B. B. Policicchio, T. He, J. Kristoff, E. Cornell, G. S. Haret-Richter, A. Trichel, R. M. Ribeiro, R. Tracy, C. Wilson, A. L. Landay, C. Apetrei, Antibiotic and antiinflammatory therapy transiently reduces inflammation and hypercoagulation in acutely SIV-infected pigtailed macaques. PLOS Pathog. 12, e1005384 (2016).

50. Á. H. Borges, M. J. Silverberg, D. Wentworth, A. E. Grulich, G. Fätkenheuer, R. Mitsuyasu, G. Tambussi, C. A. Sabin, J. D. Neaton, J. D. Lundgren; INSIGHT SMART; ESPRIT; SILCAAT Study Groups, Predicting risk of cancer during HIV infection: The role of inflammatory and coagulation biomarkers. AIDS 27, 1433-1441 (2013).

51. J. V. Baker, J. Neuhaus, D. Duprez, L. H. Kuller, R. Tracy, W. H. Belloso, S. De Wit, F. Drummond, H. C. Lane, B. Ledergerber, J. Lundgren, D. E. Nixon, N. I. Paton, J. D. Neaton; 
INSIGHT SMART Study Group, Changes in inflammatory and coagulation biomarkers: A randomized comparison of immediate versus deferred antiretroviral therapy in patients with HIV infection. J. Acquir. Immune Defic. Syndr. 56, 36-43 (2011).

52. A. Ghattas, H. R. Griffiths, A. Devitt, G. Y. H. Lip, E. Shantsila, Monocytes in coronary artery disease and atherosclerosis: Where are we now? J. Am. Coll. Cardiol. 62, 1541-1551 (2013).

53. K. S. Rogacev, B. Cremers, A. M. Zawada, S. Seiler, N. Binder, P. Ege, G. Große-Dunker, I. Heisel, F. Hornof, J. Jeken, N. M. Rebling, C. Ulrich, B. Scheller, M. Böhm, D. Fliser, G. H. Heine, $\mathrm{CD} 14^{++} \mathrm{CD} 16^{+}$monocytes independently predict cardiovascular events: $\mathrm{A}$ cohort study of 951 patients referred for elective coronary angiography. J. Am. Coll. Cardiol. 60, 1512-1520 (2012).

54. N. T. Funderburg, D. A. Zidar, C. Shive, A. Lioi, J. Mudd, L. W. Musselwhite, D. I. Simon, M. A. Costa, B. Rodriguez, S. F. Sieg, M. M. Lederman, Shared monocyte subset phenotypes in HIV-1 infection and in uninfected subjects with acute coronary syndrome. Blood 120, 4599-4608 (2012).

55. M. G. Netea, L. A. B. Joosten, E. Latz, K. H. Mills, G. Natoli, H. G. Stunnenberg, L. A. J. O'Neill, R. J. Xavier, Trained immunity: A program of innate immune memory in health and disease. Science 352, aaf1098 (2016).

56. A. R. Tenorio, Y. Zheng, R. J. Bosch, S. Krishnan, B. Rodriguez, P. W. Hunt, J. Plants, A. Seth, C. C. Wilson, S. G. Deeks, M. M. Lederman, A. L. Landay, Soluble markers of inflammation and coagulation but not T-cell activation predict non-AIDS-defining morbid events during suppressive antiretroviral treatment. J. Infect. Dis. 210, 1248-1259 (2014)

57. I. Pandrea, C. Apetrei, J. Dufour, N. Dillon, J. Barbercheck, M. Metzger, B. Jacquelin, R. Bohm, P. A. Marx, F. Barre-Sinoussi, V. M. Hirsch, M. C. Müller-Trutwin, A. A. Lackner, R. S. Veazey, Simian immunodeficiency virus SIVagm.sab infection of Caribbean African green monkeys: A new model for the study of SIV pathogenesis in natural hosts. J. Virol. 80, 4858-4867 (2006).

58. I. Pandrea, T. Gaufin, R. Gautam, J. Kristoff, D. Mandell, D. Montefiori, B. F. Keele, R. M. Ribeiro, R. S. Veazey, C. Apetrei, Functional cure of SIVagm infection in rhesus macaques results in complete recovery of $\mathrm{CD}^{+} \mathrm{T}$ cells and is reverted by $\mathrm{CD} 8^{+}$cell depletion. PLOS Pathog. 7, e1002170 (2011).

59. X. Shi, B. Gangadharan, L. F. Brass, W. Ruf, B. M. Mueller, Protease-activated receptors (PAR1 and PAR2) contribute to tumor cell motility and metastasis. Mol. Cancer Res. 2, 395-402 (2004).

60. J. Kristoff, G. Haret-Richter, D. Ma, R. M. Ribeiro, C. Xu, E. Cornell, J. L. Stock, T. He, A. D. Mobley, S. Ross, A. Trichel, C. Wilson, R. Tracy, A. Landay, C. Apetrei, I. Pandrea, Early microbial translocation blockade reduces SIV-mediated inflammation and viral replication. J. Clin. Invest. 124, 2802-2806 (2014).
61. I. M. B. Francischetti, C. J. Oliveira, G. R. Ostera, S. B. Yager, F. Debierre-Grockiego, V. Carregaro, G. Jaramillo-Gutierrez, J. C. Hume, L. Jiang, S. E. Moretz, C. K. Lin, J. M. C. Ribeiro, C. A. Long, B. K. Vickers, R. T. Schwarz, K. B. Seydel, M. lacobelli, H. C. Ackerman, P. Srinivasan, R. B. Gomes, X. Wang, R. Q. Monteiro, M. Kotsyfakis, A. Sá-Nunes, M. Waisberg, Defibrotide interferes with several steps of the coagulationinflammation cycle and exhibits therapeutic potential to treat severe malaria. Arterioscler. Thromb. Vasc. Biol. 32, 786-798 (2012).

62. J. Pinheiro, D. Bated, Mixed-Effects Models in S and S-Plus (Springer, 2002).

Acknowledgments: We would like to thank the study participants. Funding: This study was supported by the NIH Intramural Research Program, National Institute of Allergy and Infectious Diseases, and Bench-to-Bedside award R01HL117715-10S1 (to I.S. and I.P.). Part of this project has been also funded with federal funds from the National Cancer Institute, $\mathrm{NIH}$, under contract no. HHSN261200800001E. The NHP study has also been funded in part with federal funds from the NIH (R01 HL123096 and RO1 HL117715 to I.P., R01 Al119346 to C.A., and R01Al104373 to R.M.R.). The content of this publication does not necessarily reflect the views or policies of the Department of Health and Human Services, nor does mention of trade names, commercial products, or organizations imply endorsement by the U.S. government. Author contributions: M.E.S., B.B.A., I.M.B.F., I.P., and I.S. conceived and designed the research. M.E.S., B.B.A., T.H., G.H.R., K.W.T., B.B.P., A. Singh, K.D.R., V.S., D.M., E.B.-C., C.A., R.T., and I.M.B.F. performed the experiments. M.E.S., B.B.A., T.H., C.A., R.M.R., and I.M.B.F. analyzed the data. B.B.A., A. Sher, I.M.B.F., I.P., and I.S. contributed reagents/materials/analysis tools. M.E.S., B.B.A., I.P., and I.S. wrote the first draft of the manuscript. All authors have read and approved the final version of the manuscript. B.B.A. takes major responsibility for the integrity of the work and analyses. Competing interests: I.M.B.F. is the inventor on patent application (no. 7,078,508) held by the NIH that covers Ixolaris. All other authors declare that they have no competing interests. Data and materials availability: All the data supporting the findings of the study are presented in the manuscript. See table S6 for the primary data. Ixolaris is available from I.M.B.F. under a material transfer agreement with the National Institute of Allergy and Infectious Diseases, $\mathrm{NIH}$.

Submitted 6 December 2016

Accepted 11 July 2017

Published 30 August 2017

10.1126/scitransImed.aam5441

Citation: M. E. Schechter, B. B. Andrade, T. He, G. H. Richter, K. W. Tosh, B. B. Policicchio, A. Singh, K. D. Raehtz, V. Sheikh, D. Ma, E. Brocca-Cofano, C. Apetrei, R. Tracy, R. M. Ribeiro, A. Sher, I. M. B. Francischetti, I. Pandrea, I. Sereti, Inflammatory monocytes expressing tissue factor drive SIV and HIV coagulopathy. Sci. Transl. Med. 9, eaam5441 (2017). 


\section{Science Translational Medicine}

\section{Inflammatory monocytes expressing tissue factor drive SIV and HIV coagulopathy}

Melissa E. Schechter, Bruno B. Andrade, Tianyu He, George Haret Richter, Kevin W. Tosh, Benjamin B. Policicchio, Amrit Singh, Kevin D. Raehtz, Virginia Sheikh, Dongying Ma, Egidio Brocca-Cofano, Cristian Apetrei, Russel Tracy, Ruy M. Ribeiro, Alan Sher, Ivo M. B. Francischetti, Ivona Pandrea and Irini Sereti

Sci Transl Med 9, eaam5441.

DOI: 10.1126/scitransImed.aam5441

\section{Curbing complications in chronic HIV}

HIV-infected patients who have viral suppression due to treatment are still at enhanced risk of comorbidities such as neurological or cardiovascular complications, so Schechter et al. explored how inflammation and coagulation intersect in chronic HIV. To do so, they examined monocytes that express tissue factor in samples from patients or macaques infected with SIV. These monocytes appear to be crucial to coagulopathy. Treatment with a compound isolated from tick saliva, Ixolaris, can interrupt this damaging pathway. It is possible in the future that HIV patients would be treated with Ixolaris to stem some of the side effects of chronic infection.

ARTICLE TOOLS

SUPPLEMENTARY MATERIALS

RELATED
CONTENT

REFERENCES

PERMISSIONS http://stm.sciencemag.org/content/9/405/eaam5441

http://stm.sciencemag.org/content/suppl/2017/08/28/9.405.eaam5441.DC1 http://stm.sciencemag.org/content/scitransmed/9/402/eaaf1483.full http://stm.sciencemag.org/content/scitransmed/8/358/358ra125.full http://stm.sciencemag.org/content/scitransmed/9/377/eaag1809.full $\mathrm{http}: / /$ stm.sciencemag.org/content/scitransmed/8/353/353ra112.full http://stm.sciencemag.org/content/scitransmed/9/375/eaah4923.full

This article cites 61 articles, 21 of which you can access for free http://stm.sciencemag.org/content/9/405/eaam5441\#BIBL

http://www.sciencemag.org/help/reprints-and-permissions

Use of this article is subject to the Terms of Service

Science Translational Medicine (ISSN 1946-6242) is published by the American Association for the Advancement of Science, 1200 New York Avenue NW, Washington, DC 20005. 2017 (c) The Authors, some rights reserved; exclusive licensee American Association for the Advancement of Science. No claim to original U.S. Government Works. The title Science Translational Medicine is a registered trademark of AAAS. 